LPENSL-TH-11/10

DESY-T- $11 / 10$

\title{
Long-distance behavior of temperature correlation functions in the one-dimensional Bose gas
}

\author{
K. K. Kozlowski ${ }^{1}$, J. M. Maillet ${ }^{2}$, N. A. Slavnov ${ }^{3}$
}

\begin{abstract}
We describe a Bethe ansatz based method to derive, starting from a multiple integral representation, the long-distance asymptotic behavior at finite temperature of the density-density correlation function in the interacting onedimensional Bose gas. We compute the correlation lengths in terms of solutions of non-linear integral equations of the thermodynamic Bethe ansatz type. Finally, we establish a connection between the results obtained in our approach with the correlation lengths stemming from the quantum transfer matrix method.
\end{abstract}

\footnotetext{
${ }^{1}$ DESY, Hamburg, Deutschland, karol.kajetan.kozlowski@desy.de

${ }^{2}$ Laboratoire de Physique, UMR 5672 du CNRS, ENS Lyon, France, maillet@ens-lyon.fr

${ }^{3}$ Steklov Mathematical Institute, Moscow, Russia, nslavnov@mi.ras.ru
} 


\section{Introduction}

This article is devoted to the study the long-distance asymptotic behavior of the correlation functions in the one-dimensional Bose-gas at finite temperature. The method of analysis we apply builds on the method developed in the work [1. There, the long-distance asymptotic behavior of the zero temperature correlation functions of massless quantum integrable models was derived in the framework of the algebraic Bethe ansatz. This setting allows one to present the correlation functions at zero temperature as series of multiple integrals [2, 3, 4, 5, 6]. In the long-distance regime, each of the multiple integrals appearing in one of these series can be evaluated asymptotically. The resulting asymptotic series can then be summed up [1].

In [1, we have focused on the example of the generating function for correlation functions of the third components of local spin in the $X X Z$ spin-1/2 Heisenberg chain. We have mentioned, however, that the method can be applied to other integrable models as well. In particular we showed how one can use the same approach for the model of one-dimensional bosons described by the quantum nonlinear Schrödinger equation (QNLS model) using [6].

We would like to stress that the algebraic Bethe ansatz approach of [1] was fit for the analysis of the asymptotic behavior of correlation functions at zero temperature. However, in some models such as the QNLS one, it can also be applied for the evaluation of the long-distance asymptotic behavior of correlation functions at finite temperature. It is this problem that we consider in the present article.

The Hamiltonian of the QNLS model is given by

$$
H=\int_{0}^{L}\left(\partial_{x} \Psi^{\dagger} \partial_{x} \Psi+c \Psi^{\dagger} \Psi^{\dagger} \Psi \Psi-h \Psi^{\dagger} \Psi\right) d x .
$$

Here $\Psi$ and $\Psi^{\dagger}$ are Bose-fields subject to canonical, equal-time commutation relations, $c$ is a coupling constant and $h$ the chemical potential. We focus on the case of the repulsive regime (i.e. $c>0)$ and consider the model on a finite interval $[0, L]$ subject to periodic boundary conditions. We will take the thermodynamic limit $L \rightarrow \infty$ later on.

This model, for generic $c$, was first introduced and solved by Lieb and Liniger in [7, 8]. It can be considered as a generalization of the model of impenetrable bosons considered by Girardeau [9]. The spectrum of this model can be obtained by the algebraic Bethe ansatz [10, 11, 12]. The thermodynamics of the QNLS model were first studied in [13] and this analysis was made rigorous in [14. It was shown there that the state of thermal equilibrium is described by a non-linear integral equation for the excitation energy $\varepsilon(\lambda)$

$$
\varepsilon(\lambda)=\lambda^{2}-h-\frac{T}{2 \pi} \int_{\mathbb{R}} K(\lambda-\mu) \log \left(1+e^{-\frac{\varepsilon(\mu)}{T}}\right) d \mu,
$$

where $T$ is the temperature and

$$
K(\lambda)=\frac{2 c}{\lambda^{2}+c^{2}}
$$


Below we refer to equation (1.2) as the Yang-Yang equation. The state of the thermal equilibrium is given by a Dirac sea filled with a certain density of particles $\rho_{p}(\lambda)$ and an associated density of holes $\rho_{h}(\lambda)$. The total density is denoted $\rho_{t}(\lambda)=\rho_{p}(\lambda)+\rho_{h}(\lambda)$. The Fermi weight $\vartheta(\lambda)$, as usual, is defined as the ratio of the density of particles to the total density, namely,

$$
\frac{\rho_{p}(\lambda)}{\rho_{t}(\lambda)}=\vartheta(\lambda)=\left(1+e^{\frac{\varepsilon(\lambda)}{T}}\right)^{-1}
$$

and the functions $\rho_{p}(\lambda)$ and $\rho_{t}(\lambda)$ are related to each other by the integral equation

$$
\rho_{t}(\lambda)-\frac{1}{2 \pi} \int_{\mathbb{R}} K(\lambda, \mu) \rho_{p}(\mu) d \mu=\frac{1}{2 \pi} .
$$

The temperature dependent correlation functions are defined in a standard way

$$
\langle\mathcal{O}\rangle_{T}=\frac{\operatorname{tr}\left(\mathcal{O} e^{-H / T}\right)}{\operatorname{tr}\left(e^{-H / T}\right)}=\frac{\sum\langle\Omega|\mathcal{O}| \Omega\rangle e^{-E / T}}{\sum e^{-E / T}} .
$$

Here $|\Omega\rangle$ are eigenstates of the Hamiltonian (1.1) and $E$ are their eigenvalues. The sums in 1.6 are taken over the complete set of the eigenstates $|\Omega\rangle$.

Exact representations for the temperature dependent correlation functions of the QNLS model where obtained for the impenetrable Bose gas $(c=\infty)$ in [15, 16, 17] by using the free fermion structure of the model. The long-distance asymptotic behavior of the two-point functions in the impenetrable Bose gas has been derived in [18, 19, 20] with the use of RiemannHilbert problem method. The case of general coupling constant $0<c<+\infty$ was considered in [21] by the algebraic Bethe ansatz. There a series of multiple integrals was obtained for thermal density-density correlation function of the QNLS model. The method of dual fields was applied in [16, 22, 23, 24] for the derivation of determinant-type representations for various correlation functions. Those representations were used in [25, 26] for the asymptotic analysis. One should also mention the method of the asymptotic analysis based on the functional integral approach [27. Yet another approach to estimate these long-distance asymptotic behavior of correlation functions at finite temperature is provided by the conformal field theory [28, 29, 30, 31] and references therein. These last two methods are, however, restricted to the low-temperature regime only.

Recently, in the work [32], the quantum transfer matrix (QTM) approach was applied to the QNLS model. We recall that, originally, this method was developed for quantum spin chains (see the nice review [33] and references therein), where one can construct the QTM $T_{q}$ explicitly. The diagonalization of the QTM gives access to its leading eigenvalue (the logarithm of which corresponds to the free energy) as well as to the subdominant ones (which in their turn give access to the correlation lengths at $T>0$ ). In the infinite size limit, these eigenvalues are expressed as weighted integrals involving a counting function which satisfies a thermodynamic Bethe ansatz (TBA) non-linear integral equation (see also [34]). To the best of our knowledge, 
the analog of $T_{q}$ in the QNLS model is not known nowadays. Therefore, in [32], the last model was treated as some special, continuous limit of the XXZ spin chain. It was shown that, in this limit, the non-linear integral equation describing the maximal eigenvalue of the QTM goes to the Yang-Yang equation. This observation allowed the authors of [32] to obtain a multiple integral representations for the temperature correlation functions in the QNLS model.

The first steps of our method are close to the ones adopted in [21. Namely, we build on the arguments given in [35] (see also [21, 12]): in the thermodynamic limit, the representation (1.6) can be replaced, at least for local or quasi-local operators $\mathcal{O}$, by a single expectation value

$$
\langle\mathcal{O}\rangle_{T}=\frac{\left\langle\Omega_{T}|\mathcal{O}| \Omega_{T}\right\rangle}{\left\langle\Omega_{T} \mid \Omega_{T}\right\rangle},
$$

where $\left|\Omega_{T}\right\rangle$ is any one of the eigenstates of the Hamiltonian corresponding to the thermal equilibrium. This representation constitutes the starting point for our calculations. The matter is that the algebraic Bethe ansatz provides multiple integral representations for the expectation values of a wide class of operators with respect to an arbitrary eigenstate of the Hamiltonian. In particular, one can obtain such representations for the expectation values with respect to the state of thermal equilibrium. In this way, we recast $\langle\mathcal{O}\rangle_{T}$ as a series of multiple integrals. Once this is done, it remains to evaluate the asymptotic behavior (in the distance) of the obtained integrals. It is remarkable that our results are given in terms of solutions to TBA non-linear integral equations. These can be understood as the limiting (in the sense of [32]) equations describing the sub-leading eigenvalues of the QTM. Thus, our method provides a link between the method based on the representation (1.7) and the QTM approach. In particular, we obtain an extension of the QTM-based results [37, 38] for the correlation lengths in the XXZ spin chain here for the QNLS model. By applying the scaling proposed in [32] one can actually map the correlation lengths obtained for the XXZ spin chain to the ones obtained by our asymptotic analysis.

We have already mentioned that the representation for temperature correlation functions of QNLS model as a series of multiple integrals were obtained also in [21. Later it was analyzed in [39, 40, 41]. However the authors of those works restricted themselves to the analysis of the first few terms of the series. This led them to the wrong conjecture for the long-distance asymptotic behavior of the correlation functions. We would like to stress that the series of multiple integrals obtained in [21, as well as ours are not well ordered in respect to the $x \rightarrow \infty$ limit. That is to say all the summands in both series contribute to the leading asymptotic behavior as well as to the corrections. Our method allows us to compute all such contributions and re-sum them.

This article is organized as follows. In section 2, we state the problem to solve and the main results of this paper. In section 3 we derive a series of multiple integral representation for the generating function of the density-density correlation function at finite temperature. In section 4 we perform the asymptotic analysis of the individual multiple integrals appearing in the series. We re-sum these asymptotic expressions in section 5 by the use of Lagrange series. Then, in section 6 we obtain the long-distance asymptotic behavior of the density-density correlation 
function. There, we discuss the leading term and connect the various correlation lengths we obtain with the quantum transfer matrix method. In appendix A we present a numerical analysis of the expressions for the correlation lengths that we have obtained. Technical aspects relative to the formulae for the amplitudes are gathered in appendix $B$. We discuss certain integral identities in appendix C. Finally, in appendix $\mathrm{D}$ we briefly review the results we need on continuous Lagrange series.

\section{The problem to solve and the main results}

To calculate the long-distance asymptotic behavior of the density-density correlation functions we consider its generating function

$$
\left\langle e^{2 \pi i \alpha \mathcal{Q}_{x}}\right\rangle_{T}=\frac{\left\langle\Omega_{T}\left|e^{2 \pi i \alpha \mathcal{Q}_{x}}\right| \Omega_{T}\right\rangle}{\left\langle\Omega_{T} \mid \Omega_{T}\right\rangle},
$$

were $\alpha$ is a complex number 1 This generating function is defined in terms of the number of particles on $[0 ; x]$ operator $\mathcal{Q}_{x}$

$$
\mathcal{Q}_{x}=\int_{0}^{x} j(z) d z, \quad \text { with } \quad j(x)=\Psi^{\dagger}(x) \Psi(x)
$$

Then, the density-density correlation function $\langle j(x) j(0)\rangle_{T}$ is obtained from 2.1 by

$$
\langle j(x) j(0)\rangle_{T}=\left.\frac{-1}{8 \pi^{2}} \frac{\partial^{2}}{\partial x^{2}} \frac{\partial^{2}}{\partial \alpha^{2}}\left\langle e^{2 \pi i \alpha \mathcal{Q}_{x}}\right\rangle_{T}\right|_{\alpha=0} .
$$

To describe the large- $x$ asymptotic expansion of $\left\langle e^{2 \pi i \alpha \mathcal{Q}_{x}}\right\rangle_{T}$, we first consider a set of functions $u_{i}(\lambda)$ satisfying the non-linear integral equations

$$
u_{i}(\lambda)=\lambda^{2}-h_{\alpha}-\frac{T}{2 \pi} \int_{\mathbb{R}} K(\lambda-\mu) \log \left(1+e^{-\frac{u_{i}(\mu)}{T}}\right) d \lambda-i T \sum_{\ell=1}^{n}\left[\theta\left(\hat{s}_{\ell}^{+}-\lambda\right)-\theta\left(\hat{s}_{\ell}^{-}-\lambda\right)\right],
$$

where $n=0,1, \ldots$, the kernel $K(\lambda-\mu)$ is given by (1.3), $h_{\alpha}=h+2 \pi i \alpha T$, and

$$
\theta(\lambda)=i \log \left(\frac{i c+\lambda}{i c-\lambda}\right), \quad \theta^{\prime}(\lambda)=K(\lambda)
$$

The parameters $\left\{\hat{s}_{\ell}^{ \pm}\right\}$belong to the upper (resp. lower) half-plane and are the roots of the equation

$$
1+e^{-u_{i}\left(\hat{s}_{\ell}^{ \pm}\right) / T}=0
$$

\footnotetext{
${ }^{1}$ We draw the reader's attention to the fact that the combination $2 \pi i \alpha$ was denoted by $\beta$ in 1 . We did not use this notation here so as to avoid a confusion with the inverse temperature, that is traditionally denoted by $\beta$.
} 
The subscript $i$ in $u_{i}$ serves as a way to label all the possible choices of $n \operatorname{roots}\left\{\hat{s}_{\ell}^{ \pm}\right\}_{i}$. In the framework of our analysis, the roots $\hat{s}_{\ell}^{ \pm}$arise as deformations of the poles $r_{\ell}^{ \pm}$of the Fermi weight (1.4). Namely, there exist functions $\hat{s}_{\ell}^{ \pm}(\gamma)$ of some parameter $\gamma$ such that $\hat{s}_{\ell}^{ \pm}(0)=r_{\ell}^{ \pm}$ and $\hat{s}_{\ell}^{ \pm}(1)=\hat{s}_{\ell}^{ \pm}$. Thus, one can associate every set $\left\{\hat{s}_{\ell}^{ \pm}\right\}$with its pre-image $\left\{r_{\ell}^{ \pm}\right\}$. Every choice of the $\left\{r_{\ell}^{ \pm}\right\}_{i}$ uniquely yields the image $\left\{\hat{s}_{\ell}^{ \pm}\right\}_{i}$ and the corresponding function $u_{i}(\lambda)$. Thus, the subscript $i$ in the equation (2.4), in fact, enumerates different subsets of the Fermi weight poles $\left\{r_{\ell}^{ \pm}\right\}_{i}$. Note that, for a given function $u_{i}(\lambda)$, there may exist other points $w_{p} \neq \hat{s}_{\ell}^{ \pm}$such that $1+e^{-u_{i}\left(w_{p}\right) / T}=0$. Following the terminology of the QTM-based approach, we call such roots hole-type solutions.

Equation 2.4 differs from the Yang-Yang equation (1.2) by a shift of the chemical potential and the term depending on the functions $\theta\left(\hat{s}_{\ell}^{ \pm}-\lambda\right)$. Due to $(2.6)$, one can get rid of this term by changing the integration contour. Namely, let the contour $\hat{\mathcal{C}}_{i}$ be a deformation of the real axis such that moving from $\mathbb{R}$ to $\hat{\mathcal{C}}_{i}$ one only crosses the roots $\left\{\hat{s}_{\ell}^{ \pm}\right\}_{i}$ while all other solutions $w_{p}$ as well as all the poles of the Fermi weight $r_{\ell}^{ \pm}$are not crossed (see Fig. 1). Then, equation (2.4) turns into

$$
u_{i}(\lambda)=\lambda^{2}-h_{\alpha}-\frac{T}{2 \pi} \int_{\hat{\mathcal{C}}_{i}} K(\lambda-\mu) \log \left(1+e^{-\frac{u_{i}(\mu)}{T}}\right) d \lambda,
$$

and has the form of the Yang-Yang equation up to the shift of the chemical potential and the change of the integration contour. Hence, different functions $u_{i}(\lambda)$ are enumerated by different contours $\hat{\mathcal{C}}_{i}: u_{i}(\lambda)=u\left(\lambda, \hat{\mathcal{C}}_{i}\right)$. It is interesting to notice that this method of recasting equation (2.4) into (2.7) is very similar to the one used in [42] to generate higher excited states within the TBA equations by analytic continuation.

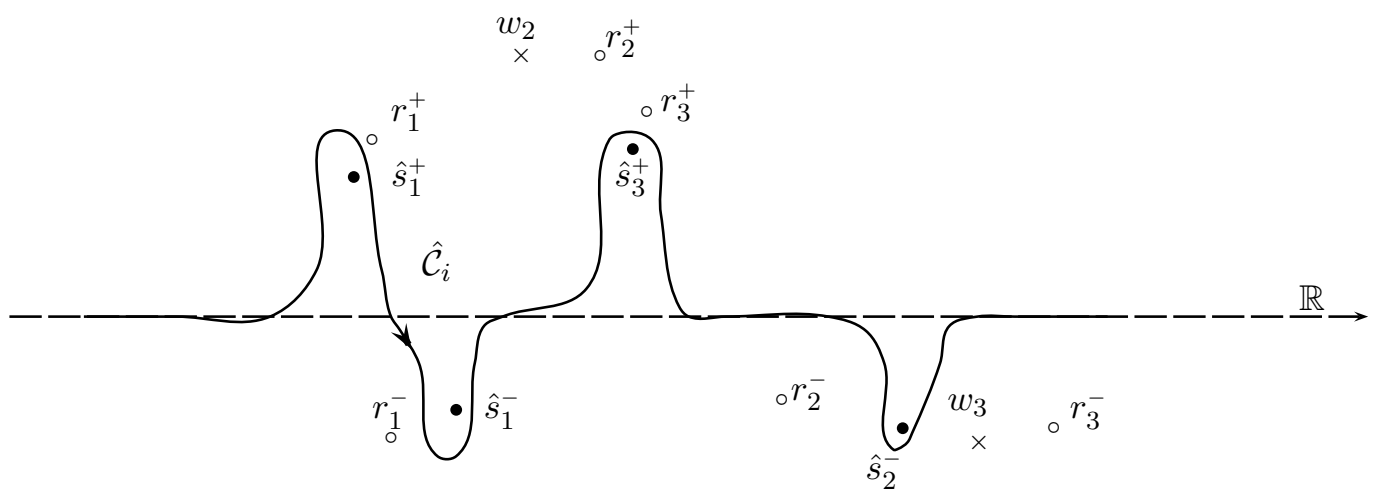

Figure 1: The poles of the Fermi weight $r_{\ell}^{ \pm}$are denoted $\circ$, the zeros $\hat{s}_{\ell}^{ \pm}$by $\bullet$, the hole-type solutions $w_{p}$ by $\times$. The integration contour $\hat{\mathcal{C}}_{i}$ bypasses the points $\hat{s}_{1}^{+}, \hat{s}_{3}^{+}$in the upper half-plane from above and bypasses the points $\hat{s}_{1}^{-}, \hat{s}_{2}^{-}$in the lower half-plane from below avoiding the points $r_{i}^{+}$and $w_{i}$. 
The large $x$ asymptotic expansion of $\left\langle e^{2 \pi i \alpha \mathcal{Q}_{x}}\right\rangle_{T}$ has the following form

$$
\left\langle e^{2 \pi i \alpha \mathcal{Q}_{x}}\right\rangle_{T} \rightarrow \sum_{i} e^{-x p_{i}} B\left[u_{i}\right], \quad x \rightarrow \infty
$$

where

$$
p_{i}=-\frac{1}{2 \pi} \int_{\hat{\mathcal{C}}_{i}} \log \left(\frac{1+e^{-\frac{u_{i}(\lambda)}{T}}}{1+e^{-\frac{\varepsilon(\lambda)}{T}}}\right) d \lambda,
$$

and the constant in $x$ amplitudes $B\left[u_{i}\right]$ are functionals of $u_{i}(\lambda)$. Their explicit but rather cumbersome form is given in section 6. It follows from (2.3) that the correlation lengths $p_{i}$ at $\alpha=0$ drive the long-distance exponential behavior of the two-point function. Our numerical computations ( $c f$ appendix A) together with the low-temperature expansions [47] confirm that given $\alpha=0$ and any $i$, i.e. for any solution $u_{i}$ to $(2.7), \Re\left(p_{i}\right) \geq 0$ and even $p_{i}=0$ if $\hat{\mathcal{C}}_{i}=\mathbb{R}$ and $\Re\left(p_{i}\right)>0$ for all other contours $\hat{\mathcal{C}}_{i} \neq \mathbb{R}$.

The sum in (2.8) is taken with respect to all possible solutions $u_{i}(\lambda)$, or what is equivalent, with respect to all different contours $\hat{\mathcal{C}}_{i}$ including $\hat{\mathcal{C}}_{i}=\mathbb{R}$. In the last case, the function $u_{i}(\lambda)$ is equal to the excitation energy $\varepsilon(\lambda)$ with the chemical potential shifted by $2 \pi i \alpha T$. It is precisely this term that gives the leading contribution to the asymptotic behavior of the density-density correlation function (see section 6).

In (2.8), we did not indicate the upper limit of the summation as well as the possible corrections. We postpone the discussion of these topics to section 6. Here, we would like to draw the reader's attention to the obvious analogy of our results for the correlation lengths with the ones obtained by the QTM approach for the temperature correlation functions of spin chains [33]. We stress however that our method is completely different and that it is applied directly to the QNLS model without any limiting procedure from the $X X Z$ Heisenberg chain like the one in 32 .

\section{Multiple integral representation}

The master equation [4, 6] is a single multidimensional contour integral representation valid for a wide class of integrable models. It allows one to obtain various types of series of multiple integral representations for certain correlation functions. In the case of the one-dimensional Bose gas at finite temperature, a series of multiple integral representation was obtained in [32] through a scaling limit from the one of the XXZ chain. Here, we work with another one that we obtain from [6] by literarily repeating the steps given in [1] for the case of the $X X Z$ Heisenberg spin chain.

Prior to taking the thermodynamic limit, one can describe the eigenstates $|\Omega\rangle$ of the Hamiltonian (1.1) by sets of real parameters $\Lambda=\left\{\lambda_{1}, \ldots, \lambda_{N}\right\}, N=0,1, \ldots$, i.e. $|\Omega\rangle=|\Omega(\Lambda)\rangle$. These parameters $\lambda_{j}$ are solutions to the Bethe equations [11, 12], and different sets $\Lambda$ yield different eigenstates $|\Omega(\Lambda)\rangle$. 
We remind that the master equation refers to a multidimensional contour integral representation for the normalized expectation value of the $e^{2 \pi i \alpha \mathcal{Q}_{x}}$ operator with respect to any eigenstate $|\Omega(\Lambda)\rangle$ of the Hamiltonian. Moreover, the expansion of this multidimensional contour integral representation into the aforementioned series of multiple integrals does not depend on the specific choice of the state $|\Omega(\Lambda)\rangle$. Hence, even when computing the thermal averages, one can still apply the results of [1]. Therefore,

$$
\begin{aligned}
\frac{\left\langle\Omega(\Lambda)\left|e^{2 \pi i \alpha \mathcal{Q}_{x}}\right| \Omega(\Lambda)\right\rangle}{\langle\Omega(\Lambda) \mid \Omega(\Lambda)\rangle}=\frac{1}{\operatorname{det}_{N} \Theta} \sum_{n=0}^{N} \frac{1}{n !} \oint_{\Gamma(\Lambda)} \prod_{j=1}^{n} \frac{d z_{j}}{2 \pi i} \sum_{\mu_{1}, \ldots, \mu_{n} \in \Lambda} \mathcal{F}_{n}\left(\begin{array}{c}
\{\mu\} \\
\{z\}
\end{array}\right) & \\
& \times \operatorname{det}_{n}\left(\frac{1}{z_{k}-\mu_{j}}\right) \prod_{j=1}^{n}\left(\frac{1}{2 \pi i L \tilde{\rho}\left(\mu_{j}\right)} \frac{e^{i x\left(\mu_{j}-z_{j}\right)}}{\mu_{j}-z_{j}}\right),
\end{aligned}
$$

where

$$
2 \pi L \tilde{\rho}\left(\lambda_{j}\right)=L+\sum_{a=1}^{N} K\left(\lambda_{j}-\lambda_{a}\right), \quad \Theta_{j k}=\delta_{j k}-\frac{K\left(\lambda_{j}-\lambda_{k}\right)}{2 \pi L \tilde{\rho}\left(\lambda_{k}\right)} .
$$

The functions $\mathcal{F}_{n}$ appearing in 3.1 are symmetric functions in the $n$ variables $\{z\}$ and in the $n$ variables $\{\lambda\}$. Their detailed description will be given later on. What only matters for the moment, is that these functions are holomorphic with respect to every variable in some open strip of fixed width $a$ around the real axis. As for the discrete summations, each parameter $\mu_{k}$ runs independently through the entire set $\Lambda$. The integrals over $z_{j}$ are taken with respect to a counterclockwise oriented, bounded and closed contour $\Gamma(\Lambda)$ surrounding the set $\Lambda$ in such a way that the only singularities of the integrand within the contour are the poles at $z_{j} \in \Lambda$. In particular, the contour $\Gamma(\Lambda)$ lies inside of the strip of width $a$.

In order to obtain the thermodynamic limit $N, L \rightarrow \infty, N / L=D=$ const of the expectation value of the $e^{2 \pi i \alpha \mathcal{Q}_{x}}$ operator, one starts by choosing a particular set $\Lambda$ and then introduces the density of particles defined by $\rho_{p}^{-1}\left(\lambda_{j}\right)=\lim _{N, L \rightarrow \infty} L\left(\lambda_{j+1}-\lambda_{j}\right)$. In the QNLS model, it can be shown that for sets $\Lambda$ having a physical interpretation (the ground state, excited states of finite energy above the ground state, states of thermal equilibrium) this limit always exists. The density of particles allows one to replace the discrete sums over $\mu_{k} \in \Lambda$ by integrals,

$$
\lim _{N, L \rightarrow \infty} \frac{1}{L} \sum_{\mu \in \Lambda} f(\mu)=\int_{\mathbb{R}} f(\lambda) \rho_{p}(\lambda) d \lambda .
$$

Above, we have made the assumption that $f(\lambda) \rho_{p}(\lambda)$ is integrable on $\mathbb{R}$.

We now consider the case where the state $|\Omega(\Lambda)\rangle$ corresponds to any finite $N, L$ representative of the state of thermal equilibrium $|\Omega\rangle_{T}$. Then, due to (1.5), it follows that the thermodynamic limit of the function $\tilde{\rho}(\lambda)$ coincides with the total density $\rho_{t}(\lambda)$. Respectively, the determinant of the matrix $\Theta_{j k}$ goes to the Fredholm determinant

$$
\lim _{N, L \rightarrow \infty} \operatorname{det} \Theta_{j k}=\operatorname{det}\left[I-\frac{1}{2 \pi} K^{(\varepsilon)}\right],
$$


where we used (1.4) and have introduced the kernel $K^{(\varepsilon)}(\lambda, \mu)=\vartheta(\mu) K(\lambda-\mu)$. Finally, using (3.3) we can replace the discrete sums over the set $\Lambda$ by integrals in every $n^{\text {th }}$ term of (3.1). Then agreeing upon

$$
\left\langle\left\langle e^{2 \pi i \alpha \mathcal{Q}_{x}}\right\rangle_{T}=\left\langle e^{2 \pi i \alpha \mathcal{Q}_{x}}\right\rangle_{T} \cdot \operatorname{det}\left[I-\frac{1}{2 \pi} K^{(\varepsilon)}\right],\right.
$$

we obtain

$$
\left\langle e^{2 \pi i \alpha \mathcal{Q}_{x}}\right\rangle_{T}=\sum_{n=0}^{\infty} \frac{1}{n !} \int_{\mathbb{R}} \frac{d^{n} \lambda}{(2 \pi i)^{n}} \oint_{\Gamma(\{\lambda\})} \frac{d^{n} z}{(2 \pi i)^{n}} \cdot \prod_{j=1}^{n}\left(\frac{\vartheta\left(\lambda_{j}\right) e^{i x\left(\lambda_{j}-z_{j}\right)}}{\lambda_{j}-z_{j}}\right) \cdot \mathcal{F}_{n}\left(\begin{array}{c}
\{\lambda\} \\
\{z\}
\end{array}\right) \cdot \operatorname{det}\left(\frac{1}{z_{k}-\lambda_{j}}\right),
$$

where the contour $\Gamma(\{\lambda\})$ surrounds counterclockwise the variables $\lambda_{1}, \ldots, \lambda_{n}$ avoiding any other singularity of the integrand. Observe that apart from the replacement of the discrete sums by integrals we also have replaced the finite sum over $n$ in (3.1) by the infinite series in (3.6). The question of convergence of this series will be discussed later, after the description of the functions $\mathcal{F}_{n}$ entering the representation $(3.6)$ for $\left\langle e^{2 \pi i \alpha \mathcal{Q}_{x}}\right\rangle_{T}$ will be given.

These functions depend on $2 n$ variables $\lambda_{1}, \ldots, \lambda_{n}$ and $z_{1}, \ldots, z_{n}$ and read,

$$
\mathcal{F}_{n}\left(\begin{array}{c}
\{\lambda\} \\
\{z\}
\end{array}\right)=W_{n}\left(\begin{array}{c}
\{\lambda\} \\
\{z\}
\end{array}\right) \cdot \prod_{j=1}^{n} \mathcal{V}_{n}\left(\lambda_{j} \mid \begin{array}{l}
\{\lambda\} \\
\{z\}
\end{array}\right)
$$

The explicit formulae for the functions $W_{n}$ and $\mathcal{V}_{n}$ involve the set of auxiliary functions:

$$
V_{\sigma ; n}(\mu)=\prod_{a=1}^{n} \frac{\mu-\lambda_{a}+i c \sigma}{\mu-z_{a}+i c \sigma}, \quad \sigma=0, \pm, \quad \text { and } \quad K_{\alpha}(\lambda)=\frac{1}{\lambda+i c}-\frac{e^{2 \pi i \alpha}}{\lambda-i c}
$$

Namely,

$$
\mathcal{V}_{n}\left(\mu \mid \begin{array}{l}
\{\lambda\} \\
\{z\}
\end{array}\right)=e^{2 \pi i \alpha} \frac{V_{+; n}(\mu)}{V_{-; n}(\mu)}-1
$$

The expression for $W_{n}$ is more involved. It can be represented as

$$
W_{n}\left(\begin{array}{c}
\{\lambda\} \\
\{z\}
\end{array}\right)=\widetilde{W}\left[V_{\sigma ; n}\right] \cdot \prod_{k=1}^{n} \frac{V_{-; n}\left(z_{k}\right)}{V_{-; n}\left(\lambda_{k}\right)}
$$

where

$$
\widetilde{W}\left[V_{\sigma ; n}\right]=\frac{\left(e^{2 \pi i \alpha}-1\right)^{2} \operatorname{det}\left[I+\frac{1}{2 \pi i} U^{(1)}\right] \operatorname{det}\left[I+\frac{1}{2 \pi i} U^{(2)}\right]}{\left(V_{+; n}^{-1}\left(\theta_{1}\right)-e^{2 \pi i \alpha} V_{-; n}^{-1}\left(\theta_{1}\right)\right)\left(V_{-; n}\left(\theta_{2}\right)-e^{2 \pi i \alpha} V_{+; n}\left(\theta_{2}\right)\right)} .
$$

Above appear two Fredholm determinants of integral operators whose kernels are

$$
U^{(1)}\left(w, w^{\prime}\right)=-V_{0 ; n}^{-1}(w) \cdot \frac{K_{\alpha}\left(w-w^{\prime}\right)-K_{\alpha}\left(\theta_{1}-w^{\prime}\right)}{V_{+; n}^{-1}(w)-e^{2 \pi i \alpha} V_{-; n}^{-1}(w)},
$$

and

$$
U^{(2)}\left(w, w^{\prime}\right)=V_{0 ; n}\left(w^{\prime}\right) \cdot \frac{K_{\alpha}\left(w-w^{\prime}\right)-K_{\alpha}\left(w-\theta_{2}\right)}{V_{-; n}\left(w^{\prime}\right)-e^{2 \pi i \alpha} V_{+; n}\left(w^{\prime}\right)} .
$$


These operators act on a counterclockwise oriented contour $\Gamma(\mathbb{R})$ surrounding the real axis. By definition, the only singularities of $U^{(k)}\left(w, w^{\prime}\right)$ inside of $\Gamma(\mathbb{R})$ are the zeros of $V_{0 ; n}(w)$ (resp. the poles of $\left.V_{0 ; n}\left(w^{\prime}\right)\right)$. In the following, when we will slightly deform the integration contour in (3.6), we will always keep this prescription for $\Gamma(\mathbb{R})$, making it larger, if necessary. The parameters $\theta_{1}$ and $\theta_{2}$ in (3.11)-3.13) are arbitrary complex numbers lying inside of the contour $\Gamma(\mathbb{R})$. It was proved in [1] that the overall combination appearing in the r.h.s. of (3.11) does not depend on a specific choice of these parameters.

Due to the rather complicated form of the functions $\mathcal{F}_{n}$, we are unable to provide a proof of the convergence of the series (3.6) in the case of general $c$. In fact, the situation is here quite analogous to the one occurring in the zero temperature case [1]. One can easily prove the convergence in the special case corresponding to the free fermion point $c=\infty$. Indeed, then $\mathcal{F}_{n}=\left(e^{2 \pi i \alpha}-1\right)^{n}$ and the integrals over $z_{j}$ can be taken explicitly. We obtain

$$
\left.\left\langle e^{2 \pi i \alpha \mathcal{Q}_{x}}\right\rangle_{T}\right|_{c=\infty}=\sum_{n=0}^{\infty} \frac{\left(e^{2 \pi i \alpha}-1\right)^{n}}{n !} \int_{\mathbb{R}} \operatorname{det}_{n}\left[\frac{\sin \frac{x}{2}\left(\lambda_{j}-\lambda_{k}\right)}{\pi\left(\lambda_{j}-\lambda_{k}\right)} \vartheta\left(\lambda_{k}\right)\right] \prod_{j=1}^{n} d \lambda_{j} .
$$

The series (3.14) is an expansion of the Fredholm determinant of the integral operator $I+V_{0}$ with

$$
V_{0}(\lambda, \mu)=\left(e^{2 \pi i \alpha}-1\right) \frac{\sin \frac{x}{2}(\lambda-\mu)}{\pi(\lambda-\mu)} \vartheta(\mu) .
$$

The general theory of Fredholm determinants ensures that the series (3.14 is absolutely convergent and defines an entire function of $e^{2 \pi i \alpha}$. It particular, the expansion coefficients decay faster than exponentially.

In the case $c<\infty$, the series 3.6 cannot be reduced to a simple form as in (3.14). Nevertheless, taking into account that the QNLS model with a general coupling constant can be considered as a smooth deformation of the free fermions case, we shall assume in the following that the series (3.6) is absolutely convergent.

In order to study the series (3.6) in the next sections, let us make two important remarks concerning the functions $W_{n}, \mathcal{V}_{n}$ and $\mathcal{F}_{n}$ present in (3.6). Although their precise expressions are rather cumbersome, it is easy to show that $W_{n}$ and $\mathcal{V}_{n}$ (and hence $\mathcal{F}_{n}$ ) are holomorphic functions within some multi-dimensional strip $\mathfrak{S}:\left|\Im\left(z_{j}\right)\right| \leq a,\left|\Im\left(\lambda_{j}\right)\right| \leq a, j=1, \ldots, n$. Though we can not determine explicitly the width $a$ of this strip, it is clear that the latter is temperature independent, because the Fermi weight $\vartheta(\lambda)(1.4$ is the only function present in (3.6) that depends on $T$. In the case of positive chemical potential, the poles of the Fermi weight accumulate, in the $T \rightarrow 0$ limit, on certain points of $\mathbb{R}$. Hence, there exists a crossover temperature $T_{0}$ such that, for $T<T_{0}$, there are always poles of the Fermi weight that are located inside of the strip $|\Im(\lambda)|<a$. The precise value of $T_{0}$ depends on the value of $a$ which, in its turn, is fixed by the analytic properties of $\mathcal{F}_{n}$. For instance, in the free fermion point, one has $T_{0}=+\infty$ as $\mathcal{F}_{n}=\left(e^{2 \pi i \alpha}-1\right)^{n}$. We will not study this question further. Simply, when $c<\infty$ we shall limit ourselves to the regime $T<T_{0}$. In such a case, the nearest (to the real axis) singularity of the integrand in (3.6) always correspond to the poles of the Fermi weight. 
The other important features of the functions $W_{n}$ and $\mathcal{V}_{n}$ concerns their reduction properties, namely,

$$
\left.\mathcal{V}_{n}\left(\omega \mid \begin{array}{c}
\{\lambda\} \\
\{z\}
\end{array}\right)\right|_{\lambda_{j}=z_{k}}=\mathcal{V}_{n-1}\left(\omega \mid \begin{array}{c}
\{\lambda\} \backslash \lambda_{j} \\
\{z\} \backslash z_{k}
\end{array}\right),\left.\quad W_{n}\left(\begin{array}{c}
\{\lambda\} \\
\{z\}
\end{array}\right)\right|_{\lambda_{j}=z_{k}}=W_{n-1}\left(\begin{array}{c}
\{\lambda\} \backslash \lambda_{j} \\
\{z\} \backslash z_{k}
\end{array}\right) .
$$

In the following, we will see that these reduction properties 3.16 are crucial for the resummation the series (3.6) in the asymptotic regime $x \rightarrow \infty$.

\section{Asymptotic behavior of multiple integrals}

The asymptotic analysis of the series $(3.6)$ can be done along the lines of the zero-temperature case [1]. The first step of that method consists in extracting the large- $x$ asymptotic behavior of each $2 n$-fold integral appearing in the series $(3.6)$. The main difference between the $T=0$ and $T>0$ situations is that, in the first case, the corrections to the leading terms have a power-law behavior in $x$, whereas, at $T>0$, they are exponentially small in $x$. Apart from this difference, the general strategy of the asymptotic analysis is the same with however several interesting technical simplifications in the case $T>0$. Below, we recall the general framework of our method.

The $2 n$-fold multiple integrals of interest have the form

$$
\mathcal{I}_{n}\left[\mathcal{F}_{n}\right]=\int_{\mathbb{R}} \frac{d^{n} \lambda}{(2 \pi i)^{n}} \oint_{\Gamma(\{\lambda\})} \frac{d^{n} z}{(2 \pi i)^{n}} \prod_{j=1}^{n}\left(\frac{\vartheta\left(\lambda_{j}\right) e^{i x\left(\lambda_{j}-z_{j}\right)}}{\lambda_{j}-z_{j}}\right) \cdot \operatorname{det}_{n}\left(\frac{1}{z_{j}-\lambda_{k}}\right) \cdot \mathcal{F}_{n}\left(\begin{array}{c}
\{\lambda\} \\
\{z\}
\end{array}\right),
$$

where $\mathcal{F}_{n}$ are holomorphic functions in some multi-dimensional strip $\mathfrak{S}:\left|\Im\left(z_{j}\right)\right| \leq a,\left|\Im\left(\lambda_{j}\right)\right| \leq a$, $j=1, \ldots, n, a$ being $n$-independent. The nearest to the real axis singularities of the integrand in (4.1) are the poles of the Fermi weight $\vartheta(\lambda)$.

\subsection{The Fredholm determinant as a multiple integrals generating function}

In this subsection, we briefly recall the connection between multiple integrals of the type 4.1 and Fredholm determinants of integral operators [1].

Let us assume for a moment that the functions $\mathcal{F}_{n}$ take the form of a pure product as

$$
\mathcal{F}_{n}\left(\begin{array}{l}
\{\lambda\} \\
\{z\}
\end{array}\right)=\prod_{p=1}^{n} \varphi\left(\lambda_{p}\right) e^{-g\left(z_{p}\right)},
$$

where $\varphi(\lambda)$ and $e^{-g(\lambda)}$ are holomorphic in the strip $|\Im(\lambda)| \leq a$. Then in 4.1), the integrals over the $z_{j}$ separate and can be computed by taking the residues at $z_{j}=\lambda_{j}$ and $z_{j}=\lambda_{k}$. A simple calculation leads to

$$
\mathcal{I}_{n}\left[\mathcal{F}_{n}\right]=\int_{\mathbb{R}} \operatorname{det}_{n}\left[V\left(\lambda_{j}, \lambda_{k}\right)\right] d^{n} \lambda=\left.\partial_{\gamma}^{n} \operatorname{det}_{\mathbb{R}}[I+\gamma V]\right|_{\gamma=0},
$$


where

$$
V(\lambda, \mu)=\frac{\vartheta(\lambda) F(\lambda)}{2 \pi i(\lambda-\mu)}\left(e^{\frac{i x}{2}(\lambda-\mu)+\frac{1}{2}(g(\lambda)-g(\mu))}-e^{-\frac{i x}{2}(\lambda-\mu)-\frac{1}{2}(g(\lambda)-g(\mu))}\right),
$$

and

$$
F(\lambda)=\varphi(\lambda) e^{-g(\lambda)}
$$

Thus, in 4.3), we have identified the multiple integral (4.1) as the $n^{\text {th }} \gamma$-derivative of the Fredholm determinant of the operator $I+\gamma V$ acting on $\mathbb{R}$ with the integral kernel (4.4).

The large- $x$ asymptotic behavior of the Fredholm determinant of the operator (4.4), up to $O\left(e^{-a x}\right)$ corrections, has been established in [44. This asymptotic expansion as well as any of its finite order $\gamma$-derivatives is uniform in $\gamma$ provided $\gamma$ belongs to a sufficiently small neighborhood of the origin. By taking the $n$-th $\gamma$-derivative of this asymptotic expansion at $\gamma=0$, we obtain the asymptotic behavior of 4.3 .

In the case of interest, $\mathcal{F}_{n}$ cannot be represented as pure product functions. However, one can proceed as in [1] and apply the density procedure (see [43] for more details), that is, to decompose $\mathcal{F}_{n}$ on the class of pure product functions :

$$
\mathcal{F}_{n}\left(\begin{array}{c}
\{\lambda\} \\
\{z\}
\end{array}\right)=\sum_{k=1}^{\infty} \prod_{p=1}^{n} \varphi_{k}\left(\lambda_{p}\right) e^{-g_{k}\left(z_{p}\right)},
$$

where the functions $\varphi_{k}(\lambda)$ and $e^{-g_{k}(\lambda)}$ are holomorphic in the strip $|\Im(\lambda)| \leq a$. We thus define

$$
F_{k}(\lambda)=\varphi_{k}(\lambda) e^{-g_{k}(\lambda)}
$$

and denote $V_{k}(\lambda, \mu)$ the kernel obtained from $V(\lambda, \mu)$ by the replacement $F \hookrightarrow F_{k}$ and $g \hookrightarrow g_{k}$. Then

$$
\mathcal{I}_{n}\left[\mathcal{F}_{n}\right]=\left.\sum_{k=1}^{\infty} \frac{\partial^{n}}{\partial \gamma^{n}} \operatorname{det}\left[I+\gamma V_{k}\right]\right|_{\gamma=0} .
$$

We stress that in 4.8 there are no problems with permuting the symbols of integration along $\mathbb{R}$ (contained in the Fredholm determinant) and summation over $k$ induced by the density procedure as the convergence in (4.6) holds in the supremum norm on the strip $\{|\Im(z)| \leq a\}$ and the weights $\vartheta(\lambda)$ ensure the convergence of the integrals 1 .

\footnotetext{
${ }^{1}$ We would like to point out that one could avoid the manipulation of infinite sums related to the density procedure as follows. Namely, one first considers the case of functions $\mathcal{F}_{n}$ that are represented as finite linear combinations of pure product functions and proceeds through all the steps in the re-summation below. At the end, once that the function one started with has been reconstructed, it is enough to observe that the answer is expressed in terms of a linear continuous functional defined on a dense subspace of a Banach space. Therefore, the obtained asymptotic expansion can be extended, by continuity, to more general classes of functions $\mathcal{F}_{n}$. As this does not alter the final conclusions, we chose not to insist on that point later on.
} 


\subsection{Large- $x$ asymptotic behavior of the Fredholm determinant}

We have seen that the problem of the calculation of the asymptotic behavior of the multiple integrals (4.1) boils down to the asymptotic analysis of the Fredholm determinant of the integral operator with the kernel (4.4). In this section, we present the results of this analysis [44]. Recall that the Fermi weight $\vartheta(\lambda)(1.4)$ appearing in (4.4) is a meromorphic function in the strip $|\Im(\lambda)| \leq a$ with simple poles at $\lambda=r_{j}^{ \pm}$, where $j=1, \ldots, M$. The superscripts \pm indicate that the corresponding pole lies in the upper (resp. lower) half-plane. The total number of poles $2 M$ is not essential for our analysis, however we have assumed that $M>0$. It is also important that the Fermi weight decays as a Gaussian when $\lambda \rightarrow \pm \infty$.

Concerning the functions $g(\lambda)$ and $F(\lambda)$ entering the kernel (4.4), in addition to their analyticity in the strip $|\Im(\lambda)| \leq a$ we assume that $g^{\prime}(\lambda) \vartheta(\lambda) F(\lambda)$ and $\vartheta(\lambda) F(\lambda)$ are integrable along any curve avoiding the poles of $\vartheta$ in this strip. This, in particular, means that $|\operatorname{tr} V|<\infty$.

Now we give several definitions and introduce new notations necessary for the formulation of the theorem about the large- $x$ asymptotic behavior of the Fredholm determinant $\operatorname{det}[I+\gamma V]$.

In addition to the points $r_{j}^{ \pm}$, we define the points $s_{j}^{ \pm}$such that $1+\gamma \vartheta\left(s_{j}^{ \pm}\right) F\left(s_{j}^{ \pm}\right)=0$. For $\gamma$ small enough, these points are slightly shifted in respect to the poles $r_{j}^{ \pm}$(see Fig. 2), but their overall number (equal to $2 M$ ) is the same in virtue of Rouché theorem.

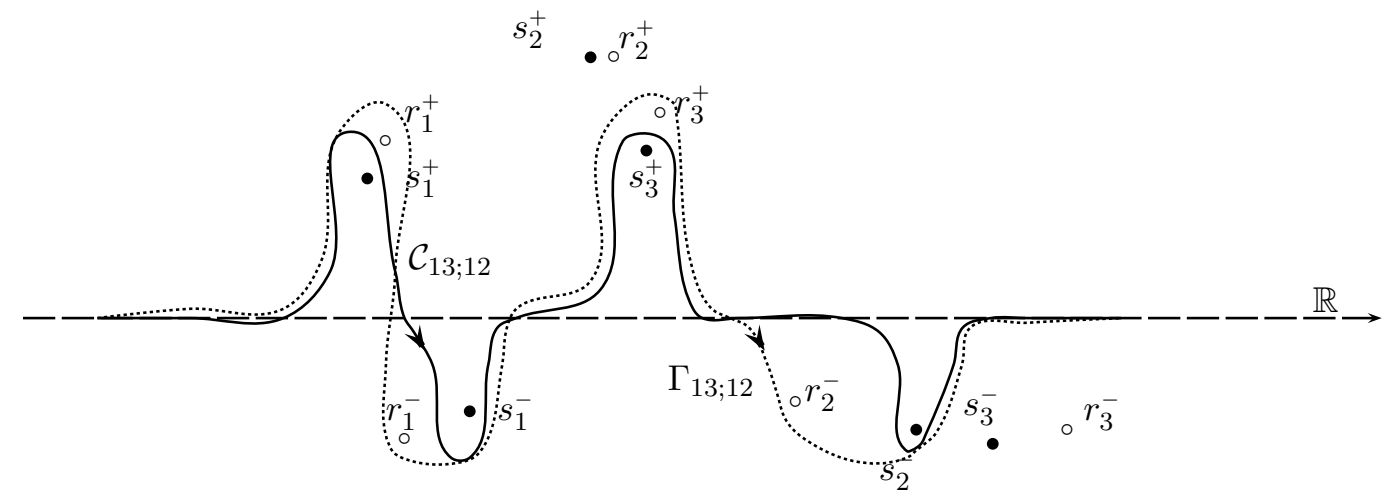

Figure 2: The poles of the Fermi weight $r_{j}^{ \pm}$are depicted by $\circ$, the zeros $s_{j}^{ \pm}$of $1+\gamma \vartheta(\lambda) F(\lambda)=0$ by $\bullet$. The integration contour $\Gamma_{13 ; 12}$ (dotted line) bypasses the points $s_{1}^{+}, s_{3}^{+}, r_{1}^{+}, r_{3}^{+}$from above and $s_{1}^{-}, s_{2}^{-}$, $r_{1}^{-}, r_{2}^{-}$from below. The contour $\mathcal{C}_{13 ; 12}$ (solid line) separates the points $s_{1}^{+}, s_{3}^{+}$from $r_{1}^{+}, r_{3}^{+}$and $s_{1}^{-}, s_{2}^{-}$ from $r_{1}^{-}, r_{2}^{-}$.

We also introduce the sets of contours $\Gamma_{\boldsymbol{j} ; \boldsymbol{k}}$ and $\mathcal{C}_{\boldsymbol{j} ; \boldsymbol{k}}$. Here $\boldsymbol{j}$ and $\boldsymbol{k}$ are multi-indices: $\boldsymbol{j}=$ $\left\{j_{1}, \ldots, j_{p}\right\}$ and $\boldsymbol{k}=\left\{k_{1}, \ldots, k_{p}\right\}$ with $1 \leq j_{s}, k_{s} \leq M$. Also $\# \boldsymbol{j}=\# \boldsymbol{k}=p$, with $p=$ $0,1, \ldots, M$. In the following, we often denote the cardinality of the sets $\boldsymbol{j}$ and $\boldsymbol{k}$ by $|\boldsymbol{j}|,|\boldsymbol{k}|$, here with $|\boldsymbol{j}|=|\boldsymbol{k}|$. 
The contour $\Gamma_{\boldsymbol{j} ; \boldsymbol{k}}$ is a deformation of the real axis such that, when moving from $\mathbb{R}$ to $\Gamma_{\boldsymbol{j} ; \boldsymbol{k}}$, one only crosses the roots $s_{\boldsymbol{j}}^{+}, s_{\boldsymbol{k}}^{-}$and the associated poles $r_{\boldsymbol{j}}^{+}, r_{\boldsymbol{k}}^{-}$, while all the other roots $s_{\ell}^{ \pm}$ and poles $r_{\ell}^{ \pm}, \ell \neq j_{a}$ or $k_{a}$, are not crossed (see Fig. 2). Note that here and below we agree upon the notation

$$
s_{\boldsymbol{j}}^{+} \equiv\left\{s_{j_{a}}^{+}\right\} \quad, \quad s_{\boldsymbol{k}}^{-} \equiv\left\{s_{k_{a}}^{+}\right\} \quad, \quad r_{\boldsymbol{j}}^{+} \equiv\left\{r_{j_{a}}^{+}\right\} \quad, \quad r_{\boldsymbol{k}}^{-} \equiv\left\{r_{k_{a}}^{-}\right\}, \quad \text { with } \quad a=1, \ldots,|\boldsymbol{j}| .
$$

Similarly, the contours $\mathcal{C}_{\boldsymbol{j} ; \boldsymbol{k}}$ are such that when moving from $\mathbb{R}$ to $\mathcal{C}_{\boldsymbol{j} ; \boldsymbol{k}}$, one only crosses the roots $s_{\boldsymbol{j}}^{+}, s_{\boldsymbol{k}}^{-}$; all poles and other roots $s_{\ell}^{ \pm}$are not crossed. In particular, $\mathcal{C}_{\boldsymbol{j} ; \boldsymbol{k}}$ separates the roots $s_{\boldsymbol{j}}^{+}, s_{\boldsymbol{k}}^{-}$from their associated poles $r_{\boldsymbol{j}}^{+}, r_{\boldsymbol{k}}^{-}$(see Fig. 2). We stress that $\mathcal{C}_{\emptyset, \emptyset}=\Gamma_{\emptyset, \emptyset}=\mathbb{R}$.

Finally we define an auxiliary function $\nu(\lambda)$ as

$$
\nu(\lambda)=\frac{-1}{2 \pi i} \log (1+\gamma \vartheta(\lambda) F(\lambda)),
$$

and a contour dependent functional

$$
\mathcal{A}_{\mathcal{L}}([g],[\nu])=-\int_{\mathcal{L}}\left(i x+g^{\prime}(\lambda)\right) \nu(\lambda) d \lambda+\iint_{\mathcal{L}} \frac{\nu(\lambda) \nu(\mu)}{\left(\lambda-\mu_{+}\right)^{2}} d \lambda d \mu,
$$

where the oriented contour $\mathcal{L}$ is either equal to $\mathcal{C}_{\boldsymbol{j} ; \boldsymbol{k}}$ or $\Gamma_{\boldsymbol{j} ; \boldsymbol{k}}$, for some $\boldsymbol{j}, \boldsymbol{k}$. In 4.11, we have stressed that the second integral is double. The symbol $\mu_{+}$means that $\mu$ is slightly shifted to the left from the oriented integration contour $\mathcal{L}$.

Theorem 4.1. 44] Let $|\gamma|$ be small enough. Then in the $x \rightarrow \infty$ limit and under the above assumptions, the Fredholm determinant of the operator $I+\gamma V$, with $V$ given by (4.4), admits the asymptotic expansion

$$
\operatorname{det}[I+\gamma V]=\sum_{\{\boldsymbol{j} ; \boldsymbol{k}\}} \exp \left(\mathcal{A}_{\mathcal{C}_{\boldsymbol{j} ; \boldsymbol{k}}}([g],[\nu])\right) \cdot\left[1+O\left(e^{-a x}\right)\right],
$$

where the sum is taken with respect to all the possible choices of multi-indices $\boldsymbol{j} ; \boldsymbol{k}$, including $\boldsymbol{j}=\emptyset$ and $\boldsymbol{k}=\emptyset$.

Remark 1. In the following, we will use the above formula 4.12 to derive the asymptotic behavior of $\mathcal{I}_{n}\left[\mathcal{F}_{n}\right]$ from the relation $(4.8)$. To do this we need to compute the $n^{\text {th }} \gamma$-derivative of the above Fredholm determinant's asymptotic behavior 4.12). In this process one can produce polynomial (in the distance $x$ ) contributions to the remainders of each term in 4.12). As a consequence the remainders will no longer be $O\left(e^{-a x}\right)$. However, they will still be a $o\left(e^{-a^{\prime} x}\right)$ for arbitrary $a^{\prime}<a$. Hence in the following the remainders in all asymptotic formulae will be given up to $o\left(e^{-a^{\prime} x}\right)$ terms for arbitrary $a^{\prime}<a$.

Remark 2. Moving all the contours $\mathcal{C}_{\boldsymbol{j} ; \boldsymbol{k}}$ in 4.12 to the real axis, one can recast the asymptotic expansion of the Fredholm determinant in the following form

$$
\operatorname{det}[I+\gamma V]=\exp \left(\mathcal{A}_{\mathbb{R}}([g],[\nu])\right) \sum_{\{\boldsymbol{j} ; \boldsymbol{k}\}} A_{\boldsymbol{j} ; \boldsymbol{k}} \prod_{b=1}^{|\boldsymbol{j}|}\left\{e^{i x\left(s_{j_{b}}^{+}-s_{k_{b}}^{-}\right)}\right\} \cdot\left[1+o\left(e^{-a^{\prime} x}\right)\right],
$$


where $A_{j ; k}$ are some constant coefficients (see [44] for their explicit form). For $\gamma$ small enough, all the roots $s_{\boldsymbol{j}}^{+}$and $s_{\boldsymbol{k}}^{-}$belong to the strip $|\Im(\lambda)|<a$. However, certain sums $\sum_{b=1}^{|\boldsymbol{j}|}\left(s_{j_{b}}^{+}-s_{k_{b}}^{-}\right)$ may have their imaginary part greater then $a$. In such a case, the corresponding terms in the expansion 4.13) (and hence, in 4.12) can be sub-leading in respect to the correction $O\left(e^{-a x}\right)$. Should such an effect occur, we will simply drop the corresponding contributions.

Remark 3. In the case of interest the parameters $a$ (and $a^{\prime}$ ) may depend on the coupling constant $c$ only. At the same time, for $\gamma$ small enough, the roots $s^{ \pm}$are close to the poles of the Fermi weight $r^{ \pm}$, whose positions depend not only on the coupling constant, but also on the temperature and the chemical potential. In particular, for $h>0$ and small $T$ all these poles (and thus, the roots $s^{ \pm}$) collapse on the real axis. Hence, for arbitrary choice of multi-indexes $\boldsymbol{j}$ and $\boldsymbol{k}$ one can always make $\Im\left(\sum_{b=1}^{|\boldsymbol{j}|}\left(s_{j_{b}}^{+}-s_{k_{b}}^{-}\right)\right)<a$ provided the temperature is small enough.

The asymptotic expansion (4.12) is uniform in $\gamma$ small enough. However, it becomes inconvenient for the calculation of the $\gamma$-derivatives of the Fredholm determinant at $\gamma=0$. Indeed, the contours $\mathcal{C}_{\boldsymbol{j} ; \boldsymbol{k}}$ are squeezed between the roots $s^{ \pm}$and the poles $r^{ \pm}$. As $s_{j}^{ \pm} \rightarrow r_{j}^{ \pm}$when $\gamma \rightarrow 0$, strictly speaking, the contours $\mathcal{C}_{\boldsymbol{j} ; \boldsymbol{k}}$ do not exist at $\gamma=0$.

A possible way to overcome this problem is to move all the contours $\mathcal{C}_{\boldsymbol{j} ; \boldsymbol{k}}$ in 4.12 to the real axis (see [44]). For our goal, it is however more convenient to deform each contour $\mathcal{C}_{\boldsymbol{j} ; \boldsymbol{k}}$ in 4.12 to its associated contour $\Gamma_{\boldsymbol{j} ; \boldsymbol{k}}$ that does exist in the above limit $\gamma \rightarrow 0$. In doing so, one crosses the poles $r_{j}^{+}$and $r_{k}^{-}$. This produces additional contributions to the functional $\mathcal{A}_{\mathcal{C}_{j ; k}}([g],[\nu])$ and makes the formula 4.12 more cumbersome.

To describe the asymptotic expansion of $\operatorname{det}[I+\gamma V]$ in terms of a sum over the contours $\Gamma_{\boldsymbol{j} ; \boldsymbol{k}}$ we introduce new notations. Let $L_{\mathcal{L}}[f](\lambda)$ be the Cauchy transform over a contour $\mathcal{L}$ of a function $f(\lambda)$ :

$$
L_{\mathcal{L}}[f](\lambda)=\int_{\mathcal{L}} \frac{f(\mu) d \mu}{\mu-\lambda} .
$$

Then the asymptotic expansion 4.12 can be written in the form

$$
\operatorname{det}[I+\gamma V]=\sum_{\{\boldsymbol{j} ; \boldsymbol{k}\}} \mathcal{U}_{\boldsymbol{j} ; \boldsymbol{k}}([g],[\nu],[F])\left[1+o\left(e^{-a^{\prime} x}\right)\right],
$$

Here, as before, the sum is taken over all the possible choices of multi-indices $\boldsymbol{j} ; \boldsymbol{k}$. The functional $\mathcal{U}_{j ; \boldsymbol{k}}$ reads

$$
\mathcal{U}_{\boldsymbol{j} ; \boldsymbol{k}}([g],[\nu],[F])=\exp \left\{-\int_{\Gamma_{\boldsymbol{j} ; \boldsymbol{k}}} g^{\prime}(\lambda) \nu(\lambda) d \lambda\right\} \prod_{a=1}^{|\boldsymbol{j}|}\left\{\gamma^{2} e^{g\left(r_{k_{a}}^{-}\right)-g\left(r_{j_{a}}^{+}\right)} F\left(r_{j_{a}}^{+}\right) F\left(r_{k_{a}}^{-}\right)\right\} \cdot U_{\boldsymbol{j} ; \boldsymbol{k}}[\nu],
$$


where

$$
\begin{aligned}
U_{\boldsymbol{j} ; \boldsymbol{k}}[\nu]=\left\{\operatorname{det}_{n}\left[\frac{1}{r_{j_{a}}^{+}-r_{k_{b}}^{-}}\right]\right\}^{2} \exp \left(\mathcal{A}_{\Gamma_{\boldsymbol{j} ; \boldsymbol{k}}}([0],[\nu])\right) & \\
& \prod_{a=1}^{|\boldsymbol{j}|}\left\{\vartheta_{r e g}\left(r_{j_{a}}^{+}\right) \vartheta_{r e g}\left(r_{k_{a}}^{-}\right) e^{i x\left(r_{k_{a}}^{-}-r_{j_{a}}^{+}\right)+2 L_{\Gamma_{j ;}}[\nu]\left(r_{k_{a}}^{-}\right)-2 L_{\Gamma_{j ;}}[\nu]\left(r_{j_{a}}^{+}\right)}\right\} .
\end{aligned}
$$

Above, we have introduced the residues of the Fermi weight $\vartheta(\lambda)$ at the poles $r_{\ell}^{ \pm}: \vartheta_{\text {reg }}\left(r_{\ell}^{ \pm}\right)=$ $\operatorname{Res}\left(\vartheta(\lambda), \lambda=r_{\ell}^{ \pm}\right)$. The detailed derivation of 4.16, 4.17) is presented in appendix C.

\subsection{Asymptotic expansion of multiple integrals}

It follows from 4.8 and the from of the expansion 4.12 , that $\mathcal{I}_{n}\left[\mathcal{F}_{n}\right]$ can be presented as

$$
\mathcal{I}_{n}\left[\mathcal{F}_{n}\right]=\sum_{\{\boldsymbol{j} ; \boldsymbol{k}\}} \mathcal{J}_{\boldsymbol{j} ; \boldsymbol{k}}\left[\mathcal{F}_{n}\right]\left[1+o\left(e^{-a^{\prime} x}\right)\right]
$$

where $\mathcal{J}_{\boldsymbol{j} ; \boldsymbol{k}}$ are some functionals that we will construct below. They can be associated with the contours $\mathcal{C}_{\boldsymbol{j} ; \boldsymbol{k}}$ (or $\Gamma_{\boldsymbol{j} ; \boldsymbol{k}}$ ), therefore we label them by multi-indices $\boldsymbol{j} ; \boldsymbol{k}$. In order to obtain their explicit form one has to perform the following steps:

- compute the $n^{\text {th }} \gamma$-derivative of $\mathcal{U}_{j ; k}([g],[\nu],[F])$ at $\gamma=0$.

- Substitute in this final expression $F \hookrightarrow F_{k}$ and $g \hookrightarrow g_{k}$.

- Take the sum over $k$ and use the decomposition (4.6) so as to express the result in terms of $\mathcal{F}_{n}$ or its partial derivatives.

We stress that the derivation of this action as given in 4.19 and 4.20 is essentially a consequence of the representation 3.7 for $\mathcal{F}_{n}$ along with the reduction properties of the functions $W_{n}$ and $\mathcal{V}_{n}$ as described in 3.16 .

Proposition 4.1. Let $\mathcal{F}_{n}$ be as given in (3.7) with $\mathcal{V}_{n}$ and $W_{n}$ satisfying to the reduction properties (3.16). Then

$$
\mathcal{J}_{j ; \boldsymbol{k}}\left[\mathcal{F}_{n}\right]=\left.\partial_{\gamma}^{n} G_{j ; \boldsymbol{k}}(\gamma)\right|_{\gamma=0}
$$

where, for $\gamma$ small enough,

$$
G_{\boldsymbol{j} ; \boldsymbol{k}}(\gamma)=\left.\sum_{m=0}^{\infty} \frac{1}{m !} \prod_{i=1}^{m} \partial_{\epsilon_{i}} \int_{\Gamma_{\boldsymbol{j} ; \boldsymbol{k}}} \prod_{i=1}^{m} \hat{\nu}\left(\lambda_{i}\right) \cdot W_{m+|\boldsymbol{j}|} \cdot \mathcal{U}_{\boldsymbol{j} ; \boldsymbol{k}}\left([0],[\hat{\nu}],\left[\mathcal{V}_{m+\mid \boldsymbol{j}]}\right]\right) d^{m} \lambda\right|_{\epsilon_{i}=0} .
$$

Here $\mathcal{U}_{\boldsymbol{j} ; \boldsymbol{k}}\left([0],[\hat{\nu}],\left[\mathcal{V}_{m+|\boldsymbol{j}|}\right]\right)$ is given by 4.16, where one should set $g(\lambda)=0$ and replace the original functions $\nu$ and $F$ by $\hat{\nu}$ and $\mathcal{V}_{m+|\boldsymbol{j}|}$ respectively. These new functions have the form

$$
\mathcal{V}_{m+|\boldsymbol{j}|}(\omega) \equiv \mathcal{V}_{m+|\boldsymbol{j}|}\left(\omega \mid \begin{array}{l}
r_{\boldsymbol{k}}^{-},\left\{\lambda_{a}\right\} \\
r_{\boldsymbol{j}}^{+},\left\{\lambda_{a}+\epsilon_{a}\right\}
\end{array}\right)
$$


and

$$
\hat{\nu}(\omega) \equiv \hat{\nu}\left(\omega \mid \begin{array}{l}
r_{\boldsymbol{k}}^{-},\left\{\lambda_{a}\right\} \\
r_{\boldsymbol{j}}^{+},\left\{\lambda_{a}+\epsilon_{a}\right\}
\end{array}\right)=\frac{-1}{2 \pi i} \log \left[1+\gamma \vartheta(\omega) \mathcal{V}_{m+|\boldsymbol{j}|}(\omega)\right]
$$

Similarly, the notation $W_{m+|j|}$ means

$$
W_{m+|\boldsymbol{j}|} \equiv W_{m+|\boldsymbol{j}|}\left(\begin{array}{l}
r_{\boldsymbol{k}}^{-},\left\{\lambda_{a}\right\} \\
r_{j}^{+},\left\{\lambda_{a}+\epsilon_{a}\right\}
\end{array}\right) .
$$

We remind here that $r_{j}=\left\{r_{j_{a}}\right\}_{a=1}^{|j|}$, etc.

Proof. The explicit form of the functional $U_{\boldsymbol{j} ; \boldsymbol{k}}[\nu]$ is given by 4.17). However, to prove Proposition 4.1, it is enough to know that it can be written in the following, quite general, form:

$$
U_{\boldsymbol{j} ; \boldsymbol{k}}[\nu]=\sum_{p=0}^{\infty} \int_{\Gamma_{\boldsymbol{j} ; \boldsymbol{k}}} U_{p}\left(\xi_{1}, \ldots, \xi_{p}\right) \prod_{j=1}^{p} \nu\left(\xi_{j}\right) d \xi_{j},
$$

where $U_{p}$ are some functions or distributions. Their explicit form as well as the fact that they depend on the poles $r_{\boldsymbol{j} / \boldsymbol{k}}^{ \pm}$does not play an essential role. Substituting the above expansion into 4.16 and expanding the exponent of $\int g^{\prime}(\lambda) \nu(\lambda) d \lambda$ into a series we obtain

$$
\begin{aligned}
\mathcal{U}_{\boldsymbol{j} ; \boldsymbol{k}}([g],[\nu],[F])=\sum_{m, p=0}^{\infty} \frac{\gamma^{2|\boldsymbol{j}|}}{m !} \int_{\Gamma_{\boldsymbol{j} ; \boldsymbol{k}}} d^{m} \lambda d^{p} \xi \cdot U_{p}(\{\xi\}) & \prod_{a=1}^{m}\left\{-g^{\prime}\left(\lambda_{a}\right) \nu\left(\lambda_{a}\right)\right\} \cdot \prod_{a=1}^{p} \nu\left(\xi_{a}\right) \\
& \times \prod_{a=1}^{|\boldsymbol{j}|}\left\{e^{g\left(r_{k_{a}}^{-}\right)-g\left(r_{j_{a}}^{+}\right)} F\left(r_{j_{a}}^{+}\right) F\left(r_{k_{a}}^{-}\right)\right\}
\end{aligned}
$$

We should now differentiate 4.25 over $\gamma$. The $n^{\text {th }} \gamma$-derivative at $\gamma=0$ of the above expression is non-vanishing only when $n \geq 2|\boldsymbol{j}|$, and then

$$
\begin{aligned}
\left.\frac{\partial^{n} \mathcal{U}_{j ; k}}{\partial \gamma^{n}}\right|_{\gamma=0}= & \sum_{m, p=0}^{\infty} \sum_{\{\ell\},\{q\}}^{\prime} C_{n}(\{\ell\},\{q\}) \int_{\Gamma_{j ; k}} \frac{d^{m} \lambda d^{p} \xi}{m !} U_{p}(\{\xi\}) \prod_{a=1}^{|j|}\left(e^{g\left(r_{k_{a}}^{-}\right)-g\left(r_{j_{a}}^{+}\right)} F\left(r_{j_{a}}^{+}\right) F\left(r_{k_{a}}^{-}\right)\right) \\
& \times\left.\prod_{a=1}^{m}\left(-g^{\prime}\left(\lambda_{a}\right) \vartheta^{\ell_{a}}\left(\lambda_{a}\right) F^{\ell_{a}}\left(\lambda_{a}\right) \frac{\partial^{\ell_{a}} \nu_{0}}{\partial \gamma^{\ell_{a}}}\right) \prod_{a=1}^{p}\left(\vartheta^{q_{a}}\left(\xi_{a}\right) F^{q_{a}}\left(\xi_{a}\right) \frac{\partial^{q_{a}} \nu_{0}}{\partial \gamma^{q_{a}}}\right)\right|_{\gamma=0}, \quad(4.26)
\end{aligned}
$$

where

$$
\nu_{0}=\frac{-1}{2 \pi i} \log (1+\gamma), \quad C_{n}(\{\ell\},\{q\})=(2|\boldsymbol{j}|) !(n-2|\boldsymbol{j}|) !\left(\prod_{a=1}^{m} \ell_{a} ! \prod_{a=1}^{p} q_{a} !\right)^{-1}
$$

and the symbol $\sum^{\prime}$ means that the sum over $\ell_{a} \geq 0$ and $q_{a} \geq 0$ in 4.26 is constrained by $\sum_{a=1}^{m} \ell_{a}+\sum_{a=1}^{p} q_{a}=n-2|\boldsymbol{j}|$. 
We have brought the result of the action of the $n^{\text {th }} \gamma$-derivative into a form convenient for the application of the density procedure. We first substitute

$$
F(\omega) \hookrightarrow \varphi_{k}(\omega) e^{-g_{k}(\omega)}, \quad g^{\prime}(\omega) \hookrightarrow g_{k}^{\prime}(\omega)
$$

in (4.26). Then the sum over $k$ is computed due to the identity

$$
\begin{aligned}
& \sum_{k=1}^{\infty} \prod_{a=1}^{m}\left\{\varphi_{k}^{\ell_{a}}\left(\lambda_{a}\right) e^{-\ell_{a} g_{k}\left(\lambda_{a}\right)}\left[-g_{k}^{\prime}\left(\lambda_{a}\right)\right]\right\} \prod_{a=1}^{p}\left\{\varphi_{k}^{q_{a}}\left(\xi_{a}\right) e^{-q_{a} g_{k}\left(\xi_{a}\right)}\right\} \prod_{a=1}^{|j|}\left\{\varphi_{k}\left(r_{j_{a}}^{+}\right) \varphi_{k}\left(r_{k_{a}}^{-}\right) e^{-2 g_{k}\left(r_{j_{a}}^{+}\right)}\right\} \\
& =\left.\prod_{a=1}^{m} \partial_{\epsilon_{a}} \cdot \mathcal{F}_{n}\left(\begin{array}{cll}
r_{\boldsymbol{j}}^{+}, r_{\boldsymbol{k}}^{-}, & \{\overbrace{\lambda_{a}, \ldots, \lambda_{a}}^{\ell_{a} \text { times }}\}_{a=1, \ldots, m}, & \{\overbrace{\xi_{b}, \ldots, \xi_{b}}^{q_{b} \text { times }}\}_{b=1, \ldots, p} \\
r_{\boldsymbol{j}}^{+}, & \{\lambda_{a}+\epsilon_{a}, \underbrace{\lambda_{a}, \ldots, \lambda_{a}}_{\ell_{a}-1 \text { times }}\}_{a=1, \ldots, m}, & \{\underbrace{\underbrace{}_{b}, \ldots, \xi_{b}}_{q_{b} \text { times }}\}_{b=1, \ldots, p}
\end{array}\right)\right|_{\epsilon_{a}=0} .
\end{aligned}
$$

Now, we use the representation (3.7) for $\mathcal{F}_{n}$ together with the reduction properties (3.16) of the functions $\mathcal{V}_{n}$ and $W_{n}$. This leads to

$$
\begin{aligned}
& \mathcal{F}_{n}\left(\begin{array}{lll}
r_{\boldsymbol{j}}^{+}, r_{\boldsymbol{k}}^{-}, & \{\overbrace{\lambda_{a}, \ldots, \lambda_{a}}^{\ell_{a} \text { times }}\}_{a=1, \ldots, m}, & \{\overbrace{\xi_{b}, \ldots, \xi_{b}}^{q_{b} \text { times }}\}_{b=1, \ldots, p} \\
r_{\boldsymbol{j}}^{+}, r_{\boldsymbol{j}}^{+}, & \{\lambda_{a}+\epsilon_{a}, \underbrace{\lambda_{a}, \ldots, \lambda_{a}}_{\ell_{a}, \ldots \text { times }}\}_{a=1, \ldots, m}, & \{\underbrace{\xi_{b}, \ldots, \xi_{b}}_{q_{b} \text { times }}\}_{b=1, \ldots, p}
\end{array}\right) \\
& =W_{m+|\boldsymbol{j}|} \cdot \prod_{b=1}^{m} \mathcal{V}_{m+|\boldsymbol{j}|}^{\ell_{b}}\left(\lambda_{b}\right) \cdot \prod_{b=1}^{p} \mathcal{V}_{m+|\boldsymbol{j}|}^{q_{b}}\left(\xi_{b}\right) \cdot \prod_{b=1}^{|\boldsymbol{j}|}\left\{\mathcal{V}_{m+|\boldsymbol{j}|}\left(r_{j_{b}}^{+}\right) \mathcal{V}_{m+|\boldsymbol{j}|}\left(r_{k_{b}}^{-}\right)\right\}
\end{aligned}
$$

where the functions $\mathcal{V}_{m+|\boldsymbol{j}|}$ and $W_{m+|\boldsymbol{j}|}$ should be understood as in 4.21, 4.23). Substituting this identity into (4.26) we obtain

$$
\begin{aligned}
\mathcal{J}_{\boldsymbol{j} ; \boldsymbol{k}}\left[\mathcal{F}_{n}\right]=\sum_{m, p=0}^{\infty} \frac{\partial_{\gamma}^{n}}{m !} \prod_{a=1}^{m} \partial_{\epsilon_{a}} \int_{\Gamma_{\boldsymbol{j} ; \boldsymbol{k}}} d^{m} \lambda d^{p} \xi U_{p}\left(\left\{\xi_{j}\right\}\right) W_{m+|\boldsymbol{j}|} \\
\quad \times\left.\prod_{b=1}^{|\boldsymbol{j}|}\left\{\mathcal{V}_{m+|\boldsymbol{j}|}\left(r_{j_{b}}^{+}\right) \mathcal{V}_{m+|\boldsymbol{j}|}\left(r_{k_{b}}^{-}\right)\right\} \prod_{a=1}^{m} \hat{\nu}\left(\lambda_{a}\right) \prod_{a=1}^{p} \hat{\nu}\left(\xi_{a}\right)\right|_{\substack{\gamma=0 \\
\epsilon_{a}=0}}
\end{aligned}
$$

where $\hat{\nu}(\omega)$ is given by 4.22$)$. The sum over $p$ in 4.31 produces $\mathcal{U}_{\boldsymbol{j} ; \boldsymbol{k}}\left([0],[\hat{\nu}],\left[\mathcal{V}_{m+\mid \boldsymbol{j}]}\right]\right)$ and we arrive at the statement of Proposition.

\section{$5 \quad$ Lagrange series}

We have presented the action of the functional $\mathcal{J}_{\boldsymbol{j} ; \boldsymbol{k}}$ on the function $\mathcal{F}_{n}$ as the $n^{\text {th }} \gamma$-derivative at $\gamma=0$ of the function $G_{\boldsymbol{j} ; \boldsymbol{k}}(\gamma)$ defined in terms of an infinite series 4.20 . In this section, we 
show that this series is nothing else but the continuous generalization of a Lagrange series, in complete analogy with the $T=0$ case studied in [1. It can be shown that, for $\gamma$ small enough, this series is absolutely convergent and that its sum can be expressed in terms of a solution of a non-linear integral equation. This is a difference in respect to the $T=0$ case where the integral equation was, eventually, linear.

The series 4.20 only contains the first order derivatives in respect to each $\epsilon_{a}$. Once that these derivatives are computed, one should set all $\epsilon_{a}=0$. Therefore, starting from (4.20), one can restrict to linear in $\epsilon_{a}, a=1, \ldots, m$ contributions to the functions $\hat{\nu}, \mathcal{V}_{m+|\boldsymbol{j}|}$, and $W_{m+|\boldsymbol{j}|}$. It means that all $\epsilon_{a}^{n}$ terms with $n>1$ can be dropped, while the terms $\epsilon_{a} \epsilon_{b}$ for $a \neq b$ etc should be kept. After such a linearization, the functions $\mathcal{V}_{m+|\boldsymbol{j}|}$ and $\hat{\nu}(\omega)$ go to

$$
\mathcal{V}_{m+|\boldsymbol{j}|}\left(\omega \mid \begin{array}{cc}
r_{\boldsymbol{k}}^{-} & \left\{\lambda_{a}\right\} \\
r_{\boldsymbol{j}}^{+} & \left\{\lambda_{a}+\epsilon_{a}\right\}
\end{array}\right) \hookrightarrow \Phi_{\boldsymbol{j} ; \boldsymbol{k}}\left(\omega \mid 2 \pi i \alpha-i \sum_{a=1}^{m} \epsilon_{a} K\left(\omega-\lambda_{a}\right)\right)
$$

and

$$
\hat{\nu}\left(\omega \mid \begin{array}{cc}
r_{\boldsymbol{k}}^{-} & \left\{\lambda_{a}\right\} \\
r_{\boldsymbol{j}}^{+} & \left\{\lambda_{a}+\epsilon_{a}\right\}
\end{array}\right) \hookrightarrow \phi_{\boldsymbol{j} ; \boldsymbol{k}}\left(\omega \mid 2 \pi i \alpha-i \sum_{a=1}^{m} \epsilon_{a} K\left(\omega-\lambda_{a}\right)\right)
$$

where

$$
\Phi_{\boldsymbol{j} ; \boldsymbol{k}}(\omega \mid X)=e^{X} \prod_{a=1}^{|\boldsymbol{j}|} \frac{e^{i \theta\left(\omega-r_{j_{a}}^{+}\right)}}{e^{i \theta\left(\omega-r_{k_{a}}^{-}\right)}}-1, \quad \phi_{\boldsymbol{j} ; \boldsymbol{k}}(\omega \mid X)=\frac{-1}{2 \pi i} \log \left[1+\gamma \vartheta(\omega) \Phi_{\boldsymbol{j} ; \boldsymbol{k}}(\omega \mid X)\right] .
$$

We recall that $K(\lambda)$ and $\theta(\lambda)$ are given resp. by (1.3), 2.5).

The linearization of the function $W_{m+|j|}$ is carried out in appendix $\mathrm{B}$. Even though its explicit form is involved, this matters little at this stage of our calculations. Therefore we present it in a rather symbolic form

$$
W_{m+|\boldsymbol{j}|}\left(\begin{array}{l}
r_{\boldsymbol{k}}^{-},\left\{\lambda_{a}\right\} \\
r_{\boldsymbol{j}}^{+},\left\{\lambda_{a}+\epsilon_{a}\right\}
\end{array}\right) \hookrightarrow \widetilde{\mathcal{W}}_{\boldsymbol{j} ; \boldsymbol{k}}\left(\sum_{a=1}^{m} \epsilon_{a} g_{\sigma}^{(1)}\left(\lambda_{a}\right) ; \sum_{a=1}^{m} \sum_{b=1}^{m} \epsilon_{a} \epsilon_{b} g^{(2)}\left(\lambda_{a}, \lambda_{b}\right)\right)
$$

where $g_{\sigma}^{(1)}$ (with $\sigma=0, \pm$ ) and $g^{(2)}$ are some functions. Their explicit expressions can be found in appendix B. Here, we point out that $\widetilde{\mathcal{W}}_{j ; \boldsymbol{k}}$ depend on the three functions $g_{\sigma}^{(1)}\left(\lambda_{a}\right), \sigma=0, \pm$ (cf appendix $\mathrm{B}$ ).

In all of the above notations, the subscripts $\boldsymbol{j}, \boldsymbol{k}$ indicate that the functions $\phi_{\boldsymbol{j} ; \boldsymbol{k}}, \Phi_{\boldsymbol{j} ; \boldsymbol{k}}$, and $\widetilde{\mathcal{W}}_{j ; k}$ depend on the sets of poles $r_{j}$ and $r_{\boldsymbol{k}}$. If these sets are empty, then we will omit the subscripts and write simply $\phi, \Phi$, and $\widetilde{\mathcal{W}}$.

Substituting (5.1), 5.2), and (5.4) into 4.20 we obtain

$$
\begin{aligned}
G_{\boldsymbol{j} ; \boldsymbol{k}}(\gamma) & =\sum_{m=0}^{\infty} \frac{1}{m !} \prod_{a=1}^{m} \partial_{\epsilon_{a}} \int_{\Gamma_{\boldsymbol{j} ; \boldsymbol{k}}} \prod_{a=1}^{m} \phi_{\boldsymbol{j} ; \boldsymbol{k}}\left(\lambda_{a} \mid 2 \pi i \alpha-i \sum_{b=1}^{m} \epsilon_{b} K\left(\lambda_{a}-\lambda_{b}\right)\right) \\
& \times\left.\widetilde{\mathcal{W}}_{\boldsymbol{j} ; \boldsymbol{k}}\left(\sum_{a=1}^{m} \epsilon_{a} g_{\sigma}^{(1)}\left(\lambda_{a}\right) ; \sum_{a=1}^{m} \sum_{b=1}^{m} \epsilon_{a} \epsilon_{b} g^{(2)}\left(\lambda_{a}, \lambda_{b}\right)\right) \mathcal{U}_{\boldsymbol{j} ; \boldsymbol{k}}\left([0],\left[\phi_{\boldsymbol{j} ; \boldsymbol{k}}\right],\left[\Phi_{\boldsymbol{j} ; \boldsymbol{k}}\right]\right) d^{m} \lambda\right|_{\epsilon_{a}=0} .
\end{aligned}
$$


The above series is the continuous generalization of the Lagrange series ( $c f$ [45] for the scalar case, 46] for the multi-dimensional case, [1] and Appendix $D$ for the continuous generalization). This series is absolutely convergent if there exists a $R_{0}>0$ such that for all $\lambda \in \Gamma_{\boldsymbol{j} ; \boldsymbol{k}}$

$$
\left|\frac{1}{2 \pi} \log \left(1+|\gamma \vartheta(\lambda)| \cdot\left|\prod_{j=1}^{n} \frac{e^{i \theta\left(\lambda-r_{j_{a}}^{+}\right)}}{e^{i \theta\left(\lambda-r_{k_{a}}^{-}\right)}} \cdot e^{2 i \pi \alpha} e^{R_{0} \int_{\Gamma_{j ; k}}|K(\lambda-\omega)| d \omega}-1\right|\right)\right|<R_{0} .
$$

This clearly holds for $\gamma$ small enough and hence the series (5.5) is then absolutely convergent. In this case, the result of summation reads (see appendix D)

$$
\begin{aligned}
G_{\boldsymbol{j} ; \boldsymbol{k}}(\gamma)=\frac{\mathcal{U}_{\boldsymbol{j} ; \boldsymbol{k}}\left([0],\left[z_{\boldsymbol{j} ; \boldsymbol{k}}\right],\left[\left(e^{-2 i \pi z_{\boldsymbol{j} ; \boldsymbol{k}}}-1\right) /(\gamma \vartheta)\right]\right)}{\operatorname{det}_{\Gamma_{\boldsymbol{j} ; \boldsymbol{k}}}\left[I+\mathcal{K}_{\boldsymbol{j} ; \boldsymbol{k}}\right]} \\
\quad \times \widetilde{\mathcal{W}}_{\boldsymbol{j} ; \boldsymbol{k}}\left(\int_{\Gamma_{\boldsymbol{j} ; \boldsymbol{k}}} g_{\sigma}^{(1)}(\lambda) z_{\boldsymbol{j} ; \boldsymbol{k}}(\lambda) d \lambda ; \int_{\Gamma_{\boldsymbol{j} ; \boldsymbol{k}}} g^{(2)}(\lambda, \mu) z_{\boldsymbol{j} ; \boldsymbol{k}}(\lambda) z_{\boldsymbol{j} ; \boldsymbol{k}}(\mu) d \lambda d \mu\right) .
\end{aligned}
$$

The function $z_{\boldsymbol{j} ; \boldsymbol{k}}(\lambda)$ appearing above is the unique solution to the non-linear integral equation

$$
z_{\boldsymbol{j} ; \boldsymbol{k}}(\lambda)-\phi_{\boldsymbol{j} ; \boldsymbol{k}}\left(\lambda \mid 2 \pi i \alpha-i \int_{\Gamma_{\boldsymbol{j} ; \boldsymbol{k}}} K(\lambda-\omega) z_{\boldsymbol{j} ; \boldsymbol{k}}(\omega) d \omega\right)=0 .
$$

Note that, the uniqueness and existence of this solution is provided by the convergence of the Lagrange series. The integral operator $I+\mathcal{K}_{\boldsymbol{j} ; \boldsymbol{k}}$ acts on the contour $\Gamma_{\boldsymbol{j} ; \boldsymbol{k}}$ with the kernel

$$
\mathcal{K}_{\boldsymbol{j} ; \boldsymbol{k}}(\lambda, \mu)=i K(\lambda-\mu) \phi_{\boldsymbol{j} ; \boldsymbol{k}}^{\prime}\left(\lambda \mid 2 i \pi \alpha-i \int_{\Gamma_{\boldsymbol{j} ; \boldsymbol{k}}} K(\lambda-\tau) z_{\boldsymbol{j} ; \boldsymbol{k}}(\tau) d \tau\right)
$$

where

$$
\phi_{j ; \boldsymbol{k}}^{\prime}(\omega \mid X)=\partial_{X} \phi_{\boldsymbol{j} ; \boldsymbol{k}}(\omega \mid X) .
$$

It is easy to see that its Fredholm determinant coincides with the functional Jacobian of equation (5.8).

Observe that apart from the appearance of the Fredholm determinant described above, the result of the Lagrange series summation reduces to the replacement of the discrete sums involving the $\epsilon_{a}$ 's by integrals over the contour $\Gamma_{\boldsymbol{j} ; \boldsymbol{k}}$ with a weight function $z_{\boldsymbol{j} ; \boldsymbol{k}}(\lambda)$. Due to this fact, and the form of equation (5.8), one has that the original arguments $\phi_{\boldsymbol{j} ; \boldsymbol{k}}$ and $\Phi_{\boldsymbol{j} ; \boldsymbol{k}}$ of the functional $\mathcal{U}_{\boldsymbol{j} ; \boldsymbol{k}}$ are replaced by $z_{\boldsymbol{j} ; \boldsymbol{k}}$ and $\left.\left(e^{-2 i \pi z_{j ; \boldsymbol{k}}}-1\right) /(\gamma \vartheta)\right)$ respectively (taking into account that $\Phi_{\boldsymbol{j} ; \boldsymbol{k}}=$ $\left.\left(e^{-2 i \pi \phi_{j} ; k}-1\right) /(\gamma \vartheta)\right)$.

The obtained result can be slightly simplified by deforming the integration contours $\Gamma_{\boldsymbol{j} ; \boldsymbol{k}}$. Indeed, it follows straightforwardly from (5.8), 5.3) that $e^{-2 \pi i z_{j ; k}(\lambda)}$ has simple poles at $r_{j}^{+}$and $r_{\boldsymbol{k}}^{-}$. Define also the sets of zeros $\hat{s}_{\boldsymbol{j}}^{+}$and $\hat{s}_{\boldsymbol{k}}^{-}$of $e^{-2 \pi i z_{j ; \boldsymbol{k}}(\lambda)}$ such that:

$$
e^{-2 \pi i z_{\boldsymbol{j} ; \boldsymbol{k}}\left(\hat{s}_{j a}^{+}\right)}=0, \quad e^{-2 \pi i z_{\boldsymbol{j} ; \boldsymbol{k}}\left(\hat{s}_{k_{a}}^{-}\right)}=0, \quad \hat{s}_{\boldsymbol{j} / \boldsymbol{k}}^{ \pm} \rightarrow r_{\boldsymbol{j} / \boldsymbol{k}}^{ \pm} \quad \text { as } \quad \gamma \rightarrow 0 .
$$


We now introduce the contour $\hat{\mathcal{C}}_{\boldsymbol{j} ; \boldsymbol{k}}$. It is a deformation of the real axis such that moving from $\mathbb{R}$ to $\hat{\mathcal{C}}_{\boldsymbol{j} ; \boldsymbol{k}}$ one only crosses the roots $\hat{s}_{j_{1}}^{+}, \ldots, \hat{s}_{j_{n}}^{+}$and $\hat{s}_{k_{1}}^{-}, \ldots, \hat{s}_{k_{n}}^{-}$, while the other roots $\hat{s}_{\ell}^{ \pm}$and all the poles $r_{\ell}^{ \pm}$are not crossed (see Fig. 11. In particular, this contour separates the poles $r_{\boldsymbol{j} / \boldsymbol{k}}^{ \pm}$ from the roots $\hat{s}_{\boldsymbol{j} / \boldsymbol{k}}^{ \pm}$.

Let us shift the integration contour $\Gamma_{\boldsymbol{j} ; \boldsymbol{k}}$ to the contour $\hat{\mathcal{C}}_{\boldsymbol{j} ; \boldsymbol{k}}$ everywhere in (5.7). Observe that, for $\gamma$ small enough, the pole and zero structure of the function $e^{-2 \pi i z_{j ; k}(\lambda)}$ in a neighborhood of the contours $\Gamma_{\boldsymbol{j} ; \boldsymbol{k}}$ and $\hat{\mathcal{C}}_{\boldsymbol{j} ; \boldsymbol{k}}$ is completely analogous to the one of the function $e^{-2 i \pi \nu(\lambda)} 4.10$. This allows us to use the method described in appendix C. However, now, all the calculations should be done in a reverse order.

In particular, the equation (C.1) applied to the contours $\Gamma_{\boldsymbol{j} ; \boldsymbol{k}}$ and $\hat{\mathcal{C}}_{\boldsymbol{j} ; \boldsymbol{k}}$ and the function $z_{\boldsymbol{j} ; \boldsymbol{k}}$ gives

$$
\int_{\Gamma_{\boldsymbol{j} ; \boldsymbol{k}}} K(\lambda-\omega) z_{\boldsymbol{j} ; \boldsymbol{k}}(\omega) d \omega=\int_{\hat{\mathcal{C}}_{\boldsymbol{j} ; \boldsymbol{k}}} K(\lambda-\omega) z_{\boldsymbol{j} ; \boldsymbol{k}}(\omega) d \omega-\sum_{a=1}^{|\boldsymbol{j}|}\left[\theta\left(\lambda-r_{k_{a}}^{-}\right)-\theta\left(\lambda-r_{j_{a}}^{+}\right)\right]
$$

Substituting this into (5.8), we find that $z_{\boldsymbol{j} ; \boldsymbol{k}}(\lambda)$ solves the integral equation

$$
z_{\boldsymbol{j} ; \boldsymbol{k}}(\lambda)-\phi\left(\lambda \mid 2 \pi i \alpha-i \int_{\hat{\mathcal{C}_{j ; \boldsymbol{k}}}} K(\lambda-\omega) z_{\boldsymbol{j} ; \boldsymbol{k}}(\omega) d \omega\right)=0,
$$

(recall that $\phi(\lambda \mid X)$ is given by (5.3) with $|\boldsymbol{j}|=0$ ). The Jacobian of equation (5.8) naturally turns into the Jacobian of the equation (5.13), and hence,

$$
\underset{\Gamma_{j ; k}}{\operatorname{det}}\left[I+\mathcal{K}_{j ; k}\right]=\underset{\hat{\mathcal{C}}_{j ; k}}{\operatorname{det}}[I+\mathcal{K}]
$$

where

$$
\mathcal{K}(\lambda, \mu)=i K(\lambda-\mu) \phi^{\prime}\left(\lambda \mid 2 i \pi \alpha-i \int_{\mathcal{C}_{j ; k}} K(\lambda-\tau) z_{j ; k}(\tau) d \tau\right),
$$

and $\phi^{\prime}$ is given by 5.10 .

Formula C.1 also yields

$$
\widetilde{\mathcal{W}}_{\boldsymbol{j} ; \boldsymbol{k}}\left(\int_{\Gamma_{\boldsymbol{j} ; \boldsymbol{k}}} g_{\sigma}^{(1)}(\lambda) z_{\boldsymbol{j} ; \boldsymbol{k}}(\lambda) d \lambda ; \ldots\right)=\widetilde{\mathcal{W}}\left(\int_{\hat{\mathcal{C}}_{\boldsymbol{j} ; \boldsymbol{k}}} g_{\sigma}^{(1)}(\lambda) z_{\boldsymbol{j} ; \boldsymbol{k}}(\lambda) d \lambda ; \ldots\right),
$$

(see appendix B for more details). Finally, making the calculations of appendix $\mathrm{C}$ in the reverse order we find that

$$
\left.\mathcal{U}_{\boldsymbol{j} ; \boldsymbol{k}}\left([0],\left[z_{j ; \boldsymbol{k}}\right],\left[\left(e^{-2 i \pi z_{j ; \boldsymbol{k}}}-1\right) /(\gamma \vartheta)\right)\right]\right)=\exp \left\{\mathcal{A}_{\hat{\mathcal{C}}_{\boldsymbol{j} ; \boldsymbol{k}}}\left([0],\left[z_{\boldsymbol{j} ; \boldsymbol{k}}\right]\right)\right\}
$$

where the functional $\mathcal{A}_{\hat{\mathcal{C}}_{j ; \boldsymbol{k}}}([0],[z])$ is given by 4 .11 with $\mathcal{L}=\hat{\mathcal{C}}_{\boldsymbol{j} ; \boldsymbol{k}}$ and $g(\lambda)=0$. 
Thus, the sum of the series 4.20 for $G_{\boldsymbol{j} ; \boldsymbol{k}}(\gamma)$ takes the form $G_{\boldsymbol{j} ; \boldsymbol{k}}(\gamma)=\frac{\exp \left\{\mathcal{A}_{\hat{\mathcal{C}}_{\boldsymbol{j} ; \boldsymbol{k}}}\left([0],\left[z_{\boldsymbol{j} ; \boldsymbol{k}}\right]\right)\right\}}{\operatorname{det}_{\hat{\mathcal{C}}_{\boldsymbol{j} ; \boldsymbol{k}}}[I+\mathcal{K}]} \cdot \widetilde{\mathcal{W}}\left(\int_{\hat{\mathcal{C}}_{\boldsymbol{j} ; \boldsymbol{k}}} g_{\sigma}^{(1)}(\lambda) z_{\boldsymbol{j} ; \boldsymbol{k}}(\lambda) d \lambda ; \int_{\hat{\mathcal{C}}_{\boldsymbol{j} ; \boldsymbol{k}}} g^{(2)}(\lambda, \mu) z_{\boldsymbol{j} ; \boldsymbol{k}}(\lambda) z_{\boldsymbol{j} ; \boldsymbol{k}}(\mu) d \lambda d \mu\right)$.

We have expressed $G_{\boldsymbol{j} ; \boldsymbol{k}}(\gamma)$ as a functional of the function $z_{\boldsymbol{j} ; \boldsymbol{k}}(\lambda)$ satisfying the non-linear integral equation (5.13). We now show that this last integral equation can be transformed into a TBA-like equation. For this, we introduce a new function $u_{j ; k}(\lambda)$ :

$$
\frac{u_{\boldsymbol{j} ; \boldsymbol{k}}(\lambda)}{T}=i \int_{\hat{\mathcal{C}_{\boldsymbol{j} ; \boldsymbol{k}}}} K(\lambda-\omega) z_{\boldsymbol{j} ; \boldsymbol{k}}(\omega) d \omega-2 \pi i \alpha+\frac{\varepsilon(\lambda)}{T},
$$

where $\varepsilon(\lambda)$ solves the Yang-Yang equation (1.2). Then, equation (5.13) yields

$$
z_{\boldsymbol{j} ; \boldsymbol{k}}(\lambda)=-\frac{1}{2 \pi i} \log \left[1+\gamma \vartheta(\lambda)\left(e^{\frac{\varepsilon(\lambda)-u_{j ; \boldsymbol{k}}(\lambda)}{T}}-1\right)\right] .
$$

Multiplying both parts of 5.20 by $i K(\mu-\lambda)$ and integrating with respect to $\lambda$ over the contour $\hat{\mathcal{C}}_{\boldsymbol{j} ; \boldsymbol{k}}$, after some simple algebra, we obtain

$$
u_{\boldsymbol{j} ; \boldsymbol{k}}(\mu)-\varepsilon(\mu)+2 \pi i \alpha T=-\frac{T}{2 \pi} \int_{\hat{\mathcal{C}}_{\boldsymbol{j} ; \boldsymbol{k}}} K(\mu-\lambda) \log \left(\frac{1+\gamma e^{-\frac{u_{\boldsymbol{j} ; \boldsymbol{k}}(\lambda)}{T}}+(1-\gamma) e^{-\frac{\varepsilon(\lambda)}{T}}}{1+e^{-\frac{\varepsilon(\lambda)}{T}}}\right) d \lambda .
$$

It is easy to see that the roots $\hat{s}_{\boldsymbol{j}}^{+}$and $\hat{s}_{\boldsymbol{k}}^{-}$satisfy the equations

$$
1+\gamma \exp \left(-u_{\boldsymbol{j} ; \boldsymbol{k}}\left(\hat{s}_{\boldsymbol{j} / \boldsymbol{k}}^{ \pm}\right) / T\right)+(1-\gamma) \exp \left(-\varepsilon\left(\hat{s}_{\boldsymbol{j} / \boldsymbol{k}}^{ \pm}\right) / T\right)=0 .
$$

When deforming the contour $\hat{\mathcal{C}}_{\boldsymbol{j} ; \boldsymbol{k}}$ to the real axis in (5.21) we cross these roots, but do not cross the poles of the Fermi weight $r_{\ell}^{ \pm}$, i.e. the points where $1+\exp \left(-\varepsilon\left(r_{\ell}^{ \pm}\right) / T\right)=0$. Therefore, we obtain

$$
\begin{aligned}
u_{\boldsymbol{j} ; \boldsymbol{k}}(\mu)-\varepsilon(\mu) & +2 \pi i \alpha T=-\frac{T}{2 \pi} \int_{\mathbb{R}} K(\mu-\lambda) \log \left[1+\gamma e^{-\frac{u_{j ; k}(\lambda)}{T}}+(1-\gamma) e^{-\frac{\varepsilon(\lambda)}{T}}\right] d \lambda \\
+ & \frac{T}{2 \pi} \int_{\mathbb{R}} K(\mu-\lambda) \log \left[1+e^{-\frac{\varepsilon(\lambda)}{T}}\right] d \lambda-i T \sum_{a=1}^{n}\left[\theta\left(\hat{s}_{j_{a}}^{+}-\mu\right)-\theta\left(\hat{s}_{k_{a}}^{-}-\mu\right)\right] .
\end{aligned}
$$

Finally, using the Yang-Yang equation we arrive at

$$
\begin{aligned}
u_{\boldsymbol{j} ; \boldsymbol{k}}(\mu)=\mu^{2}-(h+2 \pi i \alpha T)-\frac{T}{2 \pi} \int_{\mathbb{R}} K(\mu-\lambda) \log [ & \left.1+\gamma e^{-\frac{u_{\boldsymbol{j} ; \boldsymbol{k}}(\lambda)}{T}}+(1-\gamma) e^{-\frac{\varepsilon(\lambda)}{T}}\right] d \lambda \\
& -i T \sum_{a=1}^{n}\left[\theta\left(\hat{s}_{j_{a}}^{+}-\mu\right)-\theta\left(\hat{s}_{k_{a}}^{-}-\mu\right)\right] .
\end{aligned}
$$


Thus, we have reduced equation 5.13 for the function $z_{\boldsymbol{j} ; \boldsymbol{k}}(\lambda)$ to equation 5.24 for the function $u_{\boldsymbol{j} ; \boldsymbol{k}}(\lambda)$. In the following, we shall consider the function $u_{\boldsymbol{j} ; \boldsymbol{k}}(\lambda)$ as the primary object, while the function $z_{\boldsymbol{j} ; \boldsymbol{k}}(\lambda)$ will be defined by 5.20 .

Let us summarize the obtained results. We have expressed the large $x$ asymptotic behavior of the multiple integrals $\mathcal{I}_{n}\left[\mathcal{F}_{n}\right]$ (4.1) in terms of the function $G_{j ; k}(\gamma)$ via 4.18, (4.19). The function $G_{\boldsymbol{j} ; \boldsymbol{k}}(\gamma)$ is given by (5.18). It is a functional of $u_{\boldsymbol{j} ; \boldsymbol{k}}(\lambda)$, the solution to the non-linear integral equation 5.24. In its turn, $u_{\boldsymbol{j} ; \boldsymbol{k}}(\lambda)$ depends on the sets of parameters $\hat{s}_{\boldsymbol{j}}^{+}$and $\hat{s}_{\boldsymbol{k}}^{-}$ satisfying to the condition $(5.22)$.

Observe that the roots $\hat{s}_{\boldsymbol{j} / \boldsymbol{k}}^{ \pm}$depend on $\gamma: \hat{s}_{\boldsymbol{j} / \boldsymbol{k}}^{ \pm}=\hat{s}_{\boldsymbol{j} / \boldsymbol{k}}^{ \pm}(\gamma)$. One can treat them as $\gamma$ deformations of the poles $r_{\boldsymbol{j} / \boldsymbol{k}}^{ \pm}$such that $\hat{s}_{\boldsymbol{j} / \boldsymbol{k}}^{ \pm}(0)=r_{\boldsymbol{j} / \boldsymbol{k}}^{ \pm}$. Similarly, the function $u_{\boldsymbol{j} ; \boldsymbol{k}}(\lambda)=$ $u_{\boldsymbol{j} ; \boldsymbol{k}}(\lambda, \gamma)$ can be considered as a $\gamma$-deformation of the function $u_{\boldsymbol{j} ; \boldsymbol{k}}(\lambda, 0)$ which satisfies equation 5.24 at $\gamma=0$ :

$$
u_{\boldsymbol{j} ; \boldsymbol{k}}(\lambda, 0)=\varepsilon(\lambda)-2 \pi i \alpha T-i T \sum_{a=1}^{n}\left[\theta\left(r_{j_{a}}^{+}-\lambda\right)-\theta\left(r_{k_{a}}^{-}-\lambda\right)\right]
$$

The above $\gamma$-deformations are analytic in $\gamma$, at least for $\gamma$ small enough.

To conclude this section we simplify the notations used above. Recall that we have originally used the multi-indices $\boldsymbol{j}$ and $\boldsymbol{k}$ in order to denote certain subsets of the Fermi weight's poles $\boldsymbol{r}_{\boldsymbol{j}}^{+}$ and $r_{\boldsymbol{k}}^{-}$. Let us enumerate all subsets of multi-indices $\{\boldsymbol{j} ; \boldsymbol{k}\}$ by one number, say $i$. Then every subset $r_{\boldsymbol{j} / \boldsymbol{k}}^{ \pm}$can be enumerated as $\left\{r^{ \pm}\right\}_{i}$. In particular, we agree upon $\left\{r^{ \pm}\right\}_{0}=\emptyset$. However, in all other respects, the order of enumeration is not essential. Given a subset $\left\{r^{ \pm}\right\}_{i}$ we can uniquely determine the roots $\left\{\hat{s}^{ \pm}\right\}_{i}$ and the corresponding function $u_{i}(\lambda)$ as analytical $\gamma$-deformations of $\left\{r^{ \pm}\right\}_{i}$ and $u_{i}(\lambda, 0)$ (5.25). In its turn, given $\left\{\hat{s}^{ \pm}\right\}_{i}$, we can define the integration contours $\hat{\mathcal{C}}_{i}$, and hence, find the function $G_{i}(\gamma)$. Thus, the asymptotic behavior of the multiple integrals (4.1) can be written in the form

$$
\mathcal{I}_{n}\left[\mathcal{F}_{n}\right]=\left.\sum_{i} \partial_{\gamma}^{n} G_{i}(\gamma)\left[1+o\left(e^{-a^{\prime} x}\right)\right]\right|_{\gamma=0},
$$

where $i$ enumerates the subsets of multi-indices $\boldsymbol{j}$ and $\boldsymbol{k}$.

We do not write explicitly the upper limit of summation over $i$ in the formula (5.26). It depends on how many poles of the Fermi weight belong to the strip $|\Im(\lambda)|<a^{\prime}$. In particular, it follows from the Remark 3 given on page 15 that for $h>0$ and $T$ small enough and arbitrary $i_{0}$ there exists $T\left(i_{0}\right)$ such that

$$
\left.\lim _{x \rightarrow \infty} e^{a^{\prime} x} \partial_{\gamma}^{n} G_{i_{0}}(\gamma)\right|_{\gamma=0}=0, \quad \text { for } \quad T<T\left(i_{0}\right) .
$$

Therefore in this case the sum in (5.26) may contain an arbitrary number of terms. 


\section{Asymptotic behavior of the correlation function}

We have presented the large $x$ asymptotic behavior of the integrals $\mathcal{I}_{n}\left[\mathcal{F}_{n}\right]$ (4.1) in the form (5.26). We should now substitute this result into the series (3.6), what leads us to

$$
\left\langle e^{2 \pi i \alpha \mathcal{Q}_{x}}\right\rangle_{T}=\left.\sum_{n=0}^{\infty} \sum_{i} \frac{1}{n !} \partial_{\gamma}^{n} G_{i}(\gamma)\right|_{\gamma=0}+\left.\sum_{n=0}^{\infty} \sum_{i} \frac{1}{n !} \partial_{\gamma}^{n} G_{i}(\gamma) \cdot o\left(e^{-a^{\prime} x}\right)\right|_{\gamma=0} .
$$

Since $G_{i}(\gamma)$ does not depend on $n$, the first series gives the Taylor expansion for $G_{i}(\gamma)$ at $\gamma=1$. Hence, this series should result in $G_{i}(1)$ provided it is convergent at $\gamma=1$. One can easily convince himself that it is true at least for the case $c=\infty$. Indeed, in this case the sum of $G_{i}(1)$ coincides with the asymptotic expansion of the Fredholm determinant (3.15) obtained in [44. Therefore considering the QNLS model with $c<\infty$ as a smooth deformation of the free fermion case we assume that this series is convergent at $\gamma=1$, and thus we obtain

$$
\left.\sum_{n=0}^{\infty} \sum_{i} \frac{1}{n !} \partial_{\gamma}^{n} G_{i}(\gamma)\right|_{\gamma=0}=\sum_{i} G_{i}(1) .
$$

Note that the convergence of the series $(6.2)$ is related to the convergence of the Lagrange series (5.5) at $\gamma=1$. This means that the analytic $\gamma$-deformations described in the end of the previous section can be continued from a vicinity of $\gamma=0$ to the point $\gamma=1$. Hence, for the computation $G_{i}(1)$ it is enough to set $\gamma=1$ in the equations for the functions $u_{i}(\lambda)$ and $z_{i}(\lambda)$. The non-linear integral equation (5.24) for $u_{i}(\lambda)$ takes the form (2.4), while the equation (5.20) for $z_{i}(\lambda)$ turns into

$$
z_{i}(\lambda)=-\frac{1}{2 \pi i} \log \left(\frac{1+e^{-\frac{u_{i}(\lambda)}{T}}}{1+e^{-\frac{\varepsilon(\lambda)}{T}}}\right) .
$$

Substituting this into the functional $\mathcal{A}_{\hat{\mathcal{C}}_{i}}\left([0],\left[z_{i}\right]\right)$ we find

$$
\mathcal{A}_{\hat{\mathcal{C}}_{i}}\left([0],\left[z_{i}\right]\right)=-i x \int_{\hat{\mathcal{C}}_{i}} z_{i}(\lambda) d \lambda+\iint_{\hat{\mathcal{C}}_{i}} \frac{z_{i}(\lambda) z_{i}(\mu)}{\left(\lambda-\mu_{+}\right)^{2}} d \lambda d \mu,
$$

and hence, every $G_{i}(1)$ is proportional to $e^{-x p_{i}}$ with $p_{i}$ given by 2.9 ). Note that the generating function $\left\langle e^{2 \pi i \alpha \mathcal{Q}_{x}}\right\rangle_{T}$ is not necessary a decreasing function of $x$ at arbitrary complex value of $\alpha$. Therefore the real parts of the obtained $p_{i}$ are not necessary non-negative. However we should have $\Re\left(p_{i}\right) \geq 0$ at $\alpha=0$, since this case describes the physical two-point correlation function $\langle j(x) j(0)\rangle_{T}$. This property is confirmed by numerical computations (cf appendix A), and the analysis of the low-temperature limit [47].

Concerning the series corresponding to the reminder in 6.1), we assume that it remains exponentially small with respect to the first term, provided the sum over $i$ in 6.2 is restricted by some $i_{0}$. Similarly to 5.26 we do not write this upper limit of summation explicitly, because we can not find its exact value for arbitrary temperature. One can show however ( $c f$ [47]) that $i_{0}$ grows as the temperature decreases. In particular, it goes to infinity when $T \rightarrow 0$. 
Thus, we reproduce the expansion 2.8

$$
\left\langle e^{2 \pi i \alpha \mathcal{Q}_{x}}\right\rangle_{T}=\sum_{i} e^{-x p_{i}} B\left[u_{i}\right]+o\left(e^{-x p_{\max }}\right), \quad x \rightarrow \infty .
$$

where $p_{\max }=\max _{i}\left(\Re\left(p_{i}\right)\right)$.

It remains to describe the constant coefficients $B\left[u_{i}\right]$ in $(2.8)$. One part of these coefficients comes from the constant term in the functional $\mathcal{A}_{\hat{\mathcal{C}}_{i}}\left([0],\left[z_{i}\right]\right)$ 6.4 . Another part is equal to the Fredholm determinant $\operatorname{det}_{\hat{\mathcal{C}}_{i}}[I+\mathcal{K}]$ appearing in equation (5.18). Observe that, at $\gamma=1$, the derivative $\phi^{\prime}$ (5.10) reads

$$
\phi^{\prime}\left(\lambda \mid \frac{\varepsilon(\lambda)-u_{i}(\lambda)}{T}\right)=-\frac{1}{2 \pi i} \frac{1}{1+e^{-\frac{u_{i}(\lambda)}{T}}} .
$$

Thus,

$$
\underset{\hat{\mathcal{C}}_{i}}{\operatorname{det}}[I+\mathcal{K}]=\operatorname{det}_{\hat{\mathcal{C}}_{i}}\left[I-\frac{1}{2 \pi} K^{\left(u_{i}\right)}\right]
$$

where the kernel $K^{\left(u_{i}\right)}$ is the analog of $K^{(\varepsilon)}$ where $\varepsilon$ has been replaced by $u_{i}$ :

$$
K^{\left(u_{i}\right)}(\lambda, \mu)=\frac{K(\lambda-\mu)}{1+e^{\frac{u_{i}(\mu)}{T}}} .
$$

This operator acts on the contour $\hat{\mathcal{C}}_{i}$ and not on $\mathbb{R}$ as it was the case for $I+K^{(\varepsilon)}$.

Finally, the most complicated part of the coefficients $B\left[u_{i}\right]$ comes from the function $\widetilde{\mathcal{W}}$ (this part of the computations is given in appendix $B$. Combining all these results we get

$$
\begin{aligned}
& B\left[u_{i}\right]=\frac{\left(e^{2 \pi i \alpha}-1\right)^{2} \exp \left(\int_{\hat{\mathcal{C}}_{i}} \frac{z_{i}(\lambda) z_{i}(\mu)}{\left(\lambda-\mu_{+}\right)^{2}} d \lambda d \mu-C_{0}\left[z_{i}, \hat{\mathcal{C}}_{i}\right]\right)}{\left[e^{L_{\hat{\mathcal{C}}_{i}}\left[z_{i}\right]\left(\theta_{1}+i c\right)}-e^{2 \pi i \alpha+L_{\hat{\mathcal{C}}_{i}}\left[z_{i}\right]\left(\theta_{1}-i c\right)}\right]\left[e^{-L_{\hat{\mathcal{C}}_{i}}\left[z_{i}\right]\left(\theta_{2}-i c\right)}-e^{2 \pi i \alpha-L_{\hat{\mathcal{C}}_{i}}\left[z_{i}\right]\left(\theta_{2}+i c\right)}\right]} \\
& \times \frac{\operatorname{det}\left[I+\frac{1}{2 \pi i} \hat{U}^{(1)}\left[z_{i}\right]\right] \operatorname{det}\left[I+\frac{1}{2 \pi i} \hat{U}^{(2)}\left[z_{i}\right]\right]}{\operatorname{det}\left[I-\frac{1}{2 \pi} K^{(\varepsilon)}\right] \operatorname{det}\left[I-\frac{1}{2 \pi} K^{\left(u_{i}\right)}\right]} .
\end{aligned}
$$

Recall that $L_{\hat{\mathcal{C}}_{i}}\left[z_{i}\right]$ is the Cauchy transform 4.14 of the function $z_{i}(\lambda)$ on the contour $\hat{\mathcal{C}}_{i}$. The functional $C_{0}$ as well as the kernels of the integral operators $\hat{U}^{(1)}\left(w, w^{\prime},\left[z_{i}\right]\right)$ and $\hat{U}^{(2)}\left(w, w^{\prime},\left[z_{i}\right]\right)$ are given in appendix B (see resp. (B.6), (B.9), (B.10) ). The latter operators act on an counterclockwise oriented contour surrounding $\hat{\mathcal{C}}_{i}$.

\section{The leading term and corrections}

Taking the second $\alpha$-derivative of the expansion $(2.8)$ for $\left\langle e^{2 \pi i \alpha \mathcal{Q}_{x}}\right\rangle_{T}$ at $\alpha=0$ we obtain the asymptotic behavior of the density-density temperature correlation function $\langle j(x) j(0)\rangle_{T}$. We show below that the leading term of this asymptotic expansion is produced by $e^{-x p_{0}}$ corresponding to the contour $\hat{\mathcal{C}_{0}}=\mathbb{R}$ while the other $p_{i}$ 's lead to sub-leading corrections. 


\subsection{The leading term}

As expected on general grounds, we obtain the following leading term of the asymptotic behavior.

Proposition 7.1. The leading term of the asymptotic behavior of the two-point function

$$
\langle j(x) j(0)\rangle_{T}=\langle j(x) j(0)\rangle_{T}^{(0)}+O\left(x^{-\infty}\right) .
$$

is given by the square of the density of particles in the state of thermal equilibrium:

$$
\langle j(x) j(0)\rangle_{T}^{(0)}=\left(\int_{\mathbb{R}} \rho_{p}(\lambda) d \lambda\right)^{2}=\langle j(0)\rangle_{T}^{2} .
$$

This contribution stems from the choice of the contour $\hat{\mathcal{C}}_{0}=\mathbb{R}$.

Proof. Setting $\hat{\mathcal{C}_{0}}=\mathbb{R}$ in 2.7 we obtain

$$
u_{0}(\mu)=\mu^{2}-h_{\alpha}-\frac{T}{2 \pi} \int_{\mathbb{R}} K(\mu-\lambda) \log \left(1+e^{-\frac{u_{0}(\lambda)}{T}}\right) d \lambda .
$$

The form of this equation coincides with the one of the Yang-Yang equation up to the shift of the chemical potential. Thus, we have

$$
u_{0}(\lambda)=\varepsilon\left(\lambda \mid h_{\alpha}\right)
$$

where we have insisted explicitly on the dependence of $\varepsilon(\lambda)$ on $h$. Substituting this into 2.9 we find

$$
p_{0}=-\frac{1}{2 \pi} \int_{\mathbb{R}} \log \left(\frac{1+e^{-\varepsilon\left(\lambda \mid h_{\alpha}\right) / T}}{1+e^{-\varepsilon(\lambda \mid h) / T}}\right) d \lambda=\frac{1}{T}\left(\mathcal{P}-\mathcal{P}_{\alpha}\right),
$$

where we have defined

$$
\mathcal{P}=\frac{T}{2 \pi} \int_{\mathbb{R}} \log \left(1+e^{-\varepsilon(\lambda \mid h) / T}\right) d \lambda .
$$

The constant $\mathcal{P}_{\alpha}$ in 7.5 corresponds to the shifted chemical potential, i.e. it is defined by (7.6) with $\varepsilon(\lambda \mid h)$ replaced by $\varepsilon\left(\lambda \mid h_{\alpha}\right)$. This result reproduces the prediction of [12]. The quantity $\mathcal{P}$ can be interpreted as the pressure in the gas, whereas $\mathcal{P}_{\alpha}$ would correspond to the pressure in the presence of a shifted complex valued chemical potential.

Thus, we see that $p_{0} \rightarrow 0$ as $\alpha \rightarrow 0$ meaning that the contribution stemming from the choice $i=0$ (that is $(\boldsymbol{j} ; \boldsymbol{k})=(\emptyset, \emptyset))$ to the density-density correlation function does not have an exponential decay. Applying the second order derivatives over $\alpha$ and $x$ to the presumed leading order term of $\left\langle e^{2 \pi i \alpha \mathcal{Q}_{x}}\right\rangle_{T}$ we obtain

$$
\langle j(x) j(0)\rangle_{T}^{(0)}=-\left.\frac{1}{8 \pi^{2}} \frac{\partial^{2}}{\partial x^{2}} \frac{\partial^{2}}{\partial \alpha^{2}} e^{-x p_{0}} B\left[u_{0}\right]\right|_{\alpha=0}=-\left.\frac{B\left[u_{0}\right]}{4 \pi^{2}}\left(\frac{\partial p_{0}}{\partial \alpha}\right)^{2}\right|_{\alpha=0},
$$


and thus, to proceed further we should find $u_{0}(\lambda)$ and $z_{0}(\lambda)$ up to linear in $\alpha$ terms. Differentiating (7.3) over $\alpha$ at $\alpha=0$ we obtain

$$
\left.\frac{\partial u_{0}(\mu)}{\partial \alpha}\right|_{\alpha=0}=-2 \pi i T+\left.\frac{1}{2 \pi} \int_{\mathbb{R}} K^{(\varepsilon)}(\mu, \lambda) \frac{\partial u_{0}(\lambda)}{\partial \alpha}\right|_{\alpha=0} d \lambda,
$$

and hence,

$$
u_{0}(\lambda)=\varepsilon(\lambda)-4 \pi^{2} i \alpha T \rho_{t}(\lambda)+O\left(\alpha^{2}\right),
$$

where $\rho_{t}(\lambda)$ is the total density satisfying the integral equation 1.5 . Equation 1.5 can be solved in terms of the resolvent $R(\lambda, \mu)$ defined by $\left(I-\frac{1}{2 \pi} K^{(\varepsilon)}\right)(I+R)=I$ :

$$
2 \pi \rho_{t}(\mu)=1+\int_{\mathbb{R}} R(\mu, \lambda) d \lambda .
$$

Substituting (7.9) into 6.3) we obtain

$$
z_{0}(\lambda)=-2 \pi \alpha \rho_{t}(\lambda) \vartheta(\lambda)+O\left(\alpha^{2}\right)
$$

and thus, due to (2.9),

$$
\left.\frac{\partial p_{0}}{\partial \alpha}\right|_{\alpha=0}=-2 \pi i \int_{\mathbb{R}} \rho_{t}(\lambda) \vartheta(\lambda) d \lambda=-2 \pi i \int_{\mathbb{R}} \rho_{p}(\lambda) d \lambda .
$$

Hence,

$$
\langle j(x) j(0)\rangle_{T}^{(0)}=\left.\left(\int_{\mathbb{R}} \rho_{p}(\lambda) d \lambda\right)^{2} \cdot B\left[u_{0}\right]\right|_{\alpha=0},
$$

and it remains to apply Lemma 7.1 in order to conclude that $B\left[u_{0}\right]=1$ at $\alpha=0$.

Lemma 7.1. $B\left[u_{0}\right]=1$ at $\alpha=0$.

Proof. Since $z_{0}(\lambda) \rightarrow 0$ at $\alpha \rightarrow 0$, we conclude that

$$
\exp \left(\int_{\mathbb{R}} \frac{z_{0}(\lambda) z_{0}(\mu)}{\left(\lambda-\mu_{+}\right)^{2}} d \lambda d \mu-C_{0}\left[z_{0}, \mathbb{R}\right]\right)=1, \quad \text { at } \quad \alpha=0 .
$$

For the calculation of the limit of the remaining part of equation 66.9), we use the obvious properties of the Cauchy transform $L_{\mathbb{R}}\left[z_{0}\right](\omega)$ :

$$
\begin{aligned}
& L_{\mathbb{R}}\left[z_{0}\right](\omega+i 0)-L_{\mathbb{R}}\left[z_{0}\right](\omega-i 0)=2 \pi i z_{0}(\omega), \\
& L_{\mathbb{R}}\left[z_{0}\right](\omega+i c)-L_{\mathbb{R}}\left[z_{0}\right](\omega-i c)=\frac{1}{T}\left(u_{0}(\lambda)-\varepsilon(\lambda)+2 \pi i \alpha T\right)=2 \pi i \alpha\left(1-2 \pi \rho_{t}(\lambda)\right)+O\left(\alpha^{2}\right) .
\end{aligned}
$$


The last property follows from (5.19). Using (7.15) we find at $\alpha \rightarrow 0$

$$
\frac{e^{2 \pi i \alpha}-1}{e^{L_{\mathbb{R}}\left[z_{0}\right]\left(\theta_{1}+i c\right)}-e^{2 \pi i \alpha+L_{\mathbb{R}}\left[z_{0}\right]\left(\theta_{1}-i c\right)}}=\frac{\left(e^{2 \pi i \alpha}-1\right) e^{-2 \pi i \alpha-L_{\mathbb{R}}\left[z_{0}\right]\left(\theta_{1}-i c\right)}}{e^{\left(u_{0}\left(\theta_{1}\right)-\varepsilon\left(\theta_{1}\right)\right) / T}-1} \rightarrow-\frac{1}{2 \pi \rho_{t}\left(\theta_{1}\right)},
$$

and

$$
\frac{e^{2 \pi i \alpha}-1}{e^{-L_{\mathbb{R}}\left[z_{0}\right]\left(\theta_{2}-i c\right)}-e^{2 \pi i \alpha-L_{\mathbb{R}}\left[z_{0}\right]\left(\theta_{2}+i c\right)}}=\frac{\left(e^{2 \pi i \alpha}-1\right) e^{-2 \pi i \alpha+L_{\mathbb{R}}\left[z_{0}\right]\left(\theta_{2}-i c\right)}}{e^{\left(u_{0}\left(\theta_{2}\right)-\varepsilon\left(\theta_{2}\right)\right) / T}-1} \rightarrow-\frac{1}{2 \pi \rho_{t}\left(\theta_{2}\right)} .
$$

Finally we should compute the ratio of determinants in the second line of (6.9). Consider, for example the determinant of the operator $I+\frac{1}{2 \pi i} U^{(1)}$. Due to the factor $e^{L_{\mathbb{R}}\left[z_{0}\right](w)}$, the kernel $\hat{U}^{(1)}\left(w, w^{\prime}\right)$ has a cut on the real axis. Hence the action of the integral operator $\hat{U}^{(1)}$ can be reduced to an action on the real axis with the kernel

$$
\left.\left.\hat{U}^{(1)}\right|_{\Gamma(\mathbb{R})} \rightarrow \tilde{U}^{(1)}\right|_{\mathbb{R}}=\left.\left(\hat{U}_{-}^{(1)}-\hat{U}_{+}^{(1)}\right)\right|_{\mathbb{R}},
$$

where $\hat{U}_{ \pm}^{(1)}$ are the limiting values of $\hat{U}^{(1)}$ from the upper (lower) half-planes. Using equations (7.15) we obtain

$$
\underset{\Gamma(\mathbb{R})}{\operatorname{det}}\left[I+\frac{1}{2 \pi i} \hat{U}^{(1)}\left[z_{0}\right]\right]=\operatorname{det}\left[I+\frac{1}{2 \pi i} \tilde{U}^{(1)}\left[z_{0}\right]\right],
$$

where the operator in the r.h.s. acts on $\mathbb{R}$ and its kernel reads

$$
\tilde{U}^{(1)}\left(w, w^{\prime}\right)=-e^{L_{\mathbb{R}}\left[z_{0}\right](w-i 0)-L_{\mathbb{R}}\left[z_{0}\right](w-i c)-2 \pi i \alpha} \frac{\left(1-e^{2 \pi i z_{0}(w)}\right)\left(K_{\alpha}\left(w-w^{\prime}\right)-K_{\alpha}\left(\theta_{1}-w^{\prime}\right)\right)}{e^{\left(u_{0}(w)-\varepsilon(w)\right) / T}-1} .
$$

Setting now $\alpha=0$ and using (7.9), (7.11) we obtain

$$
\left.\underset{\Gamma(\mathbb{R})}{\operatorname{det}}\left[I+\frac{1}{2 \pi i} \hat{U}^{(1)}\left[z_{0}\right]\right]\right|_{\alpha=0}=\operatorname{det}\left[I-\frac{1}{2 \pi}\left(K^{(\varepsilon)}\left(w, w^{\prime}\right)-K^{(\varepsilon)}\left(\theta_{1}, w^{\prime}\right)\right)\right] .
$$

Thus, in terms of the resolvent $R(\lambda, \mu)$ the ratio of determinants can be presented as

$$
\begin{aligned}
\frac{\operatorname{det}_{\mathbb{R}}\left[I-\frac{1}{2 \pi}\left(K^{(\varepsilon)}\left(w, w^{\prime}\right)-K^{(\varepsilon)}\left(\theta_{1}, w^{\prime}\right)\right)\right]}{\operatorname{det}_{\mathbb{R}}\left[I-\frac{1}{2 \pi} K^{(\varepsilon)}\left(w, w^{\prime}\right)\right]}=\operatorname{det}[I+ & \left.R\left(\theta_{1}, w^{\prime}\right)\right] \\
& =1+\int_{\mathbb{R}} R\left(\theta_{1}, w\right) d w=2 \pi \rho_{t}\left(\theta_{1}\right),
\end{aligned}
$$

where we have used (7.10). Similarly one has

$$
\left.\frac{\operatorname{det}_{\Gamma(\mathbb{R})}\left[I+\frac{1}{2 \pi i} \hat{U}^{(2)}\left[z_{0}\right]\right]}{\operatorname{det}_{\mathbb{R}}\left[I-\frac{1}{2 \pi} K^{(u)}\left(w, w^{\prime}\right)\right]}\right|_{\alpha=0}=2 \pi \rho_{t}\left(\theta_{2}\right)
$$

and we arrive at the statement of Lemma 7.1 . 


\subsection{The corrections}

It is clear that for other terms in the expansion (2.8) $u_{i}(\lambda) \neq \varepsilon(\lambda)$ even at $\alpha=0$. This yields $p_{i} \neq 0$ at $\alpha=0$ and $\hat{\mathcal{C}} \neq \mathbb{R}$. Therefore after taking the second $\alpha$-derivative and setting $\alpha=0$ the corresponding terms of the expansion contain exponential factors $e^{-x p_{i}}$. Our numerical computations together with the low-temperature limit support that the real part of the $p_{i}$ 's are indeed positive and, hence, the corresponding terms in the asymptotic expansion are subleading. In particular, in the low-temperature limit, equation (2.7) can be solved for all possible choices of subsets $\boldsymbol{j}, \boldsymbol{k}$. Respectively, one can find all $p_{i}$. It follows from this analysis, that the main sub-leading contribution to the asymptotic behavior of $\left\langle e^{2 \pi i \alpha \mathcal{Q}_{x}}\right\rangle_{T}$ originates from the contours $\hat{\mathcal{C}}_{i}$, where $\left\{\hat{s}^{ \pm}\right\}_{i}$ are $\gamma$-deformations of the poles $\left\{r^{ \pm}\right\}_{i}$ lying nearest to the real axis. We conjecture that this property holds for the finite temperature as well.

Finally, we would like to give an interpretation of the results obtained from the viewpoint of the quantum transfer matrix approach [33]. As we have mentioned already this method was developed for the description of quantum spin chains, but it was shown in 32 that in a special continuous limit it can be applied to the description of thermodynamics in the one-dimensional Bose gas. The central object of this method is a function $\mathfrak{a}_{0}(\lambda)$ constructed via certain solution of Bethe equations and satisfying a non-linear integral equation. Knowing this function allows one to calculate the maximal eigenvalue $\Lambda_{0}$ of the quantum transfer matrix $T_{q}$. In the continuous limit mentioned above, one can establish the following correspondence

$$
\begin{aligned}
& \mathfrak{a}_{0}(\lambda) \mapsto e^{-\varepsilon(\lambda) / T}, \\
& \Lambda_{0} \mapsto \frac{1}{2 \pi} \int_{\mathbb{R}} \log \left(1+e^{-\varepsilon(\lambda) / T}\right) d \lambda .
\end{aligned}
$$

The sub-leading eigenvalues $\Lambda_{i}$ of $T_{q}$ can be expressed in terms of functions $\mathfrak{a}_{i}(\lambda)$ satisfying the same type of the integral equation, but on deformed integration contours surrounding some zeros of $1+\mathfrak{a}_{i}(\lambda)$. It is easy to show that, in the continuous limit, these contours go into the contours $\hat{\mathcal{C}}_{i}$. Then one should have the following correspondence

$$
\begin{aligned}
& \mathfrak{a}_{i}(\lambda) \mapsto e^{-u_{i}(\lambda) / T}, \\
& \Lambda_{i} \mapsto \frac{1}{2 \pi} \int_{\hat{\mathcal{C}}_{i}} \log \left(1+e^{-u_{i}(\lambda) / T}\right) d \lambda .
\end{aligned}
$$

Taking into account 2.9 we obtain the following mapping

$$
\left(\frac{\Lambda_{i}\left(h_{\alpha}\right)}{\Lambda_{0}(h)}\right)^{m} \mapsto e^{-x p_{i}}
$$

where $m$ is the lattice distance that scales to the distance $x$ in the continuous limit. In 7.26 ), we have also stressed that the eigenvalue $\Lambda_{0}(h)$ corresponds to the quantum transfer matrix $T_{q}(h)$ with the chemical potential $h$, while the eigenvalues $\Lambda_{i}\left(h_{\alpha}\right)$ correspond to the quantum transfer 
matrix $T_{q}\left(h_{\alpha}\right)$ with the shifted chemical potential $h_{\alpha}$. Thus, the rates of the exponential decay in the asymptotic behavior of $\left\langle e^{\alpha \mathcal{Q}_{x}}\right\rangle_{T}$ appear to be nothing else but the ratios of the eigenvalues of the quantum transfer matrix (in the continuous limit).

\section{Conclusion}

The main goal of this article was to derive the long distance asymptotic behavior of temperature correlation functions of one-dimensional bosons in the framework of the algebraic Bethe ansatz approach. Considering the example of the two-point function of densities we showed that this asymptotic behavior can be expressed in terms of solutions of the TBA equations. This result is quite expected from the viewpoint of the QTM method.

Comparing to the QTM approach, we should say that our derivation is certainly more involved technically. However in our opinion these two methods are complementary, in particular speaking about their range of applications. Our starting point is the master equation, which is a multiple integral representation for various correlation functions, including the dynamical ones. Recall that such type of representations is known now for an arbitrary integrable model possessing the six-vertex $R$-matrix [6]. The expansion of the master equation into the series allows us to take the thermodynamic limit. Then in the asymptotic regime this series turns into the generalized Lagrange series, which can be summed up explicitly in terms of solutions to some integral equations. The last ones appear to be just the equations providing the dressing of bare variables originally entering the representation for the correlation function. We have demonstrated such a mechanism already in the case of zero temperature correlations [1]. We have now shown how it works in the case of thermal correlation functions.

Our derivation however is not free of several assumptions of technical kind. For example, such questions as the convergence of the series 3.6 and 6.2 formally remain unsolved (although these series are absolutely convergent at the free fermion point). We think nevertheless that the results obtained under these assumptions are themselves strong arguments in favor of their validity. As an additional test for our results we will consider the low-temperature limit of our asymptotic expansion in a forthcoming publication. In this limit, the non-linear integral equations determining the correlation functions in the asymptotic regime can be solved explicitly in terms of dressed physical quantities such as the energy, momentum, and charge. In particular, we will show [47] that the low-temperature limit of our results reproduces the conformal field theory predictions for the exponential decay of the correlation functions. We are however able to go well beyond these predictions as our results not only hold for all ranges of temperatures but also provide explicit formulae for the corresponding amplitudes. Moreover, it will be shown that the dependence of these amplitudes in terms of powers of the temperature in the lowtemperature limit can be computed explicitly, in complete agreement with the CFT predictions [47. 


\section{Acknowledgements}

We are very grateful to N. Kitanine and V. Terras for useful and numerous discussions. J. M. M. and N. S. are supported by CNRS. We also acknowledge the support from the GDRI-471 of CNRS "French-Russian network in Theoretical and Mathematical Physics" and RFBR-CNRS09-01-93106L-a. N. S. is also supported by the Program of RAS Mathematical Methods of the Nonlinear Dynamics, RFBR-08-01-00501a, RFBR-09-01-12150ofi-m. K. K. K. is supported by the EU Marie-Curie Excellence Grant MEXT-CT-2006-042695. N. S. and K. K. K. would like to thank the Theoretical Physics group of the Laboratory of Physics at ENS Lyon for hospitality, which makes this collaboration possible.

\section{A Numerical analysis of the correlation lengths}

The inverse correlation lengths $\Re\left(p_{i}\right)$ are defined in terms of the integral 2.9 involving the solution $u_{i}$ to the TBA non-linear integral equation (2.7). Hence, the $p_{i}$ depend on the coupling constant $c$, the temperature $T$, and the chemical potential $h$. Apart from these parameters, they also depend on the choice of the system of roots $\left\{\hat{s}^{ \pm}\right\}_{i}$ and it is to this dependence that the subscript $i$ refers to. In this appendix, we gather four plots resulting from our numerical computations of several correlation lengths (the real part of the $p_{i}$ ) as functions of their parameters.
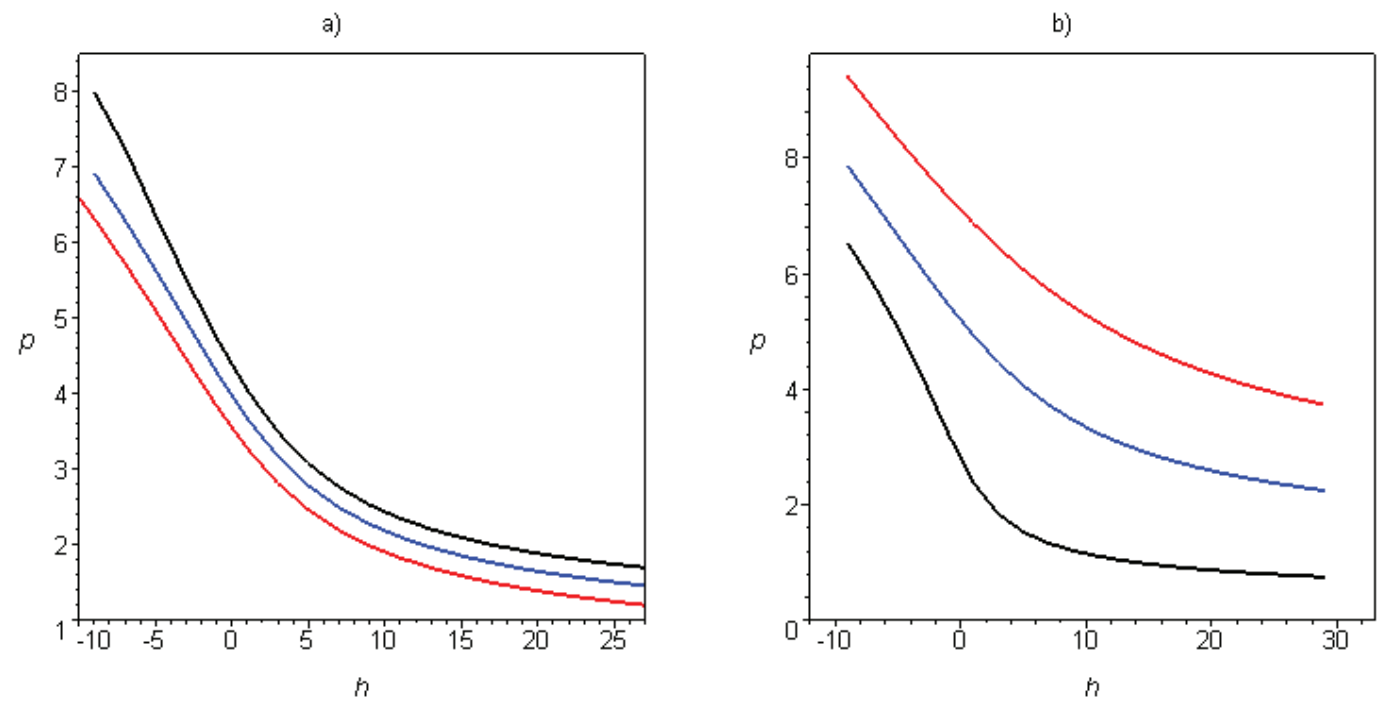

Figure 3: The dependence $p=p(h)$ in a) for a fixed temperature $T=2$ and different coupling constants $c=7$ (black), $c=10$ (blue) and $c=\infty$ (red). In b) the coupling constant is fixed at $c=10$ and the temperature is set to $T=1$ (black), $T=3$ (blue) and $T=5$ (red).

The situation considered in the first three figures (Fig. 3 a) and b), Fig. 4 a)) corresponds to the correlation length determined in terms of the solution to the non-linear integral equation 
a)

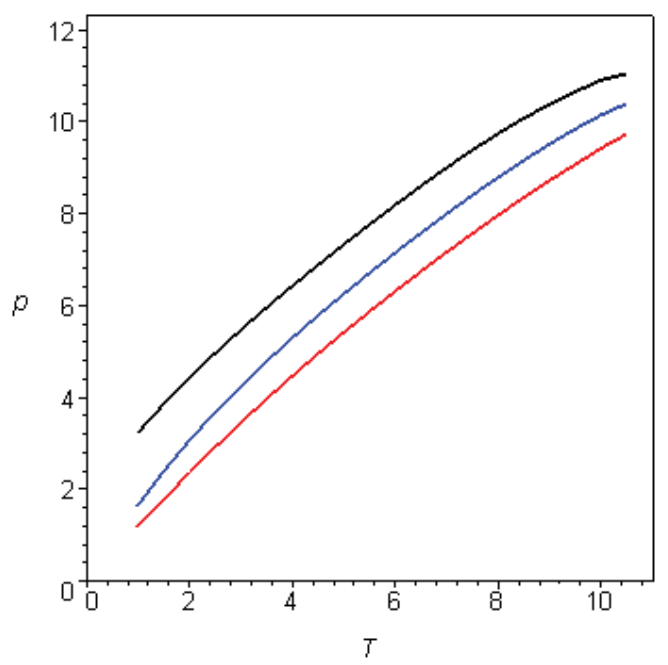

b)

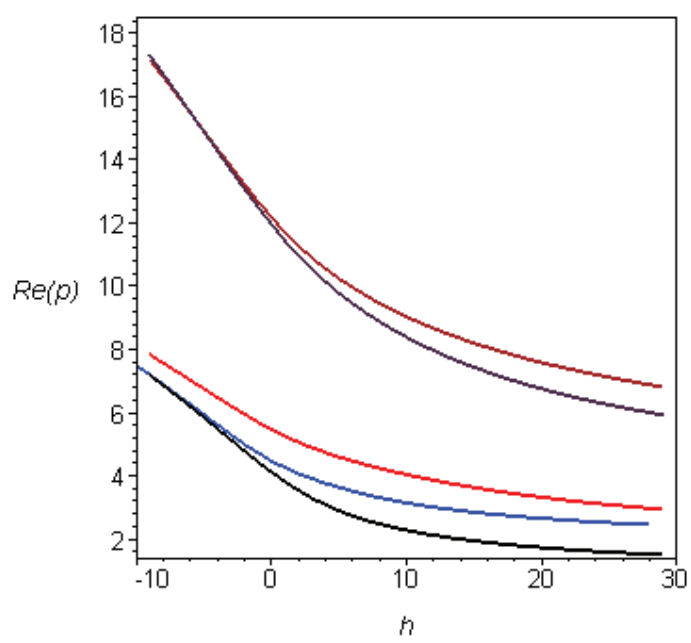

Figure 4: In a), the dependence $p=p(T)$ is presented for the coupling constant $c=10$ and at different values of the chemical potential $h=-1$ (black), $h=4$ (blue) and $h=9$ (red). In b) we have represented $\Re(p)$ as a function of the chemical potential $h$ for the coupling constant fixed to $c=10$ and the temperature to $T=2$. The three cases correspond to one pair of roots $\hat{s}^{ \pm}$in various positions : (i) $\hat{s}^{+}$is the nearest root in the first quadrant, $\hat{s}^{-}$is the nearest root in the fourth quadrant (black); (ii) $\hat{s}^{+}$ is the nearest root in the first quadrant, $\hat{s}^{-}$is the nearest root in the third quadrant (blue); (iii) $\hat{s}^{+}$is the next after the nearest root in the first quadrant, $\hat{s}^{-}$is the nearest root in the fourth quadrant (red). Two cases with two pairs of roots $\hat{s}^{ \pm}$: (iv) $\hat{s}_{1}^{ \pm}$are the nearest roots in the first and the fourth quadrants, $\hat{s}_{2}^{ \pm}$ are the next after the nearest roots in the first and the fourth quadrants (violet); (v) $\hat{s}_{1}^{ \pm}$are the nearest roots in the first and the fourth quadrants, $\hat{s}_{2}^{ \pm}$are the next after the nearest roots in the first and the third quadrants (brown).

with one root $\hat{s}^{+}$in the first quadrant of $\mathbb{C}$ and one root $\hat{s}^{-}$in the fourth quadrant. These roots are built as $\gamma$ deformations of the poles of the Fermi weight lying closest to the real axis and located in these quadrants. For this case, the corresponding $p$ is real.

On Fig. 3 a) we have represented $p$ as a function of chemical potential for a fixed temperature and several values of the coupling constant $c$. Similarly, in Fig. 3 b) we have also represented $p$ as a function of chemical potential but for a fixed value of the coupling constant $c$ and different values of the temperature. On Fig. 4 a) we have represented $p$ as a function of temperature for a fixed value of the coupling constant and several values of the chemical potential. For all these quantities, the numerical values are given in dimensionless units after proper rescaling of the TBA non-linear integral equation (2.7).

The last plot Fig. 4 b) represents different correlation lengths $\Re(p)$ as a function of the chemical potential and for a fixed value of the coupling constant and the temperature. There, we have considered several possible configurations of the system of roots $\left\{\hat{s}^{ \pm}\right\}$for which $p$ are complex numbers. 


\section{B Linearized form of $W_{m}$}

The explicit expression for the function $W_{m}$ is given by $3.10-(3.13)$. It contains Fredholm determinants whose kernels depend on the products (3.8). At $\left\{z_{k}\right\}=\left\{\lambda_{k}+\epsilon_{k}\right\}$ we have, to the linear order in each of the $\epsilon_{a}$ 's

$$
V_{\sigma}^{(m+|\boldsymbol{j}|)}(w) \hookrightarrow \prod_{a=1}^{|\boldsymbol{j}|} \frac{w-r_{k_{a}}^{-}+i c \sigma}{w-r_{j_{a}}^{+}+i c \sigma} \cdot \exp \left\{\sum_{a=1}^{m} \epsilon_{a} g_{\sigma}^{(1)}\left(\lambda_{a}\right)\right\}, \quad \sigma=0, \pm,
$$

where

$$
g_{\sigma}^{(1)}(\lambda) \equiv g_{\sigma}^{(1)}(w, \lambda)=\frac{1}{w-\lambda+i \sigma c}, \quad \sigma=0, \pm .
$$

Apart form the products of the type (B.1), the function $W_{m}$ also contains the product of the functions $V_{-}^{(m+|\boldsymbol{j}|)}$ (see (3.10)). Setting $\left\{z_{k}\right\}=\left\{\lambda_{k}+\epsilon_{k}\right\}$ we obtain

$$
\begin{aligned}
\prod_{a=1}^{m} \frac{V_{-}^{(m+|\boldsymbol{j}|)}\left(z_{a}\right)}{V_{-}^{(m+|\boldsymbol{j}|)}\left(\lambda_{a}\right)} \cdot \prod_{a=1}^{|\boldsymbol{j}|} \frac{V_{-}^{(m+|\boldsymbol{j}|)}\left(r_{j_{a}}^{+}\right)}{V_{-}^{(m+|\boldsymbol{j}|)}\left(r_{k_{a}}^{-}\right)} \hookrightarrow \\
\times \exp \left\{\sum_{a=1}^{m} \sum_{b=1}^{|\boldsymbol{j}|} \epsilon_{a}\left[g_{+}^{(1)}\left(r_{j_{b}}^{+}, \lambda_{a}\right)+g_{-}^{(1)}\left(r_{j_{b}}^{+}, \lambda_{a}\right)-g_{-}^{(1)}\left(r_{k_{b}}^{-}, \lambda_{a}\right)-g_{+}^{(1)}\left(r_{k_{b}}^{-}, \lambda_{a}\right)\right]\right\} \\
\times \prod_{a, b=1}^{|\boldsymbol{j}|} \frac{\left(r_{k_{a}}^{-}-r_{j_{b}}^{+}-i c\right)\left(r_{j_{a}}^{+}-r_{k_{b}}^{-}-i c\right)}{\left(r_{k_{a}}^{-}-r_{k_{b}}^{-}-i c\right)\left(r_{j_{a}}^{+}-r_{j_{b}}^{+}-i c\right)} \cdot \exp \left\{\sum_{a=1}^{m} \sum_{b=1}^{m} \epsilon_{a} \epsilon_{b} g^{(2)}\left(\lambda_{a}, \lambda_{b}\right)\right\},
\end{aligned}
$$

where

$$
g^{(2)}(\lambda, \mu)=-\frac{1}{(\lambda-\mu-i c)^{2}} .
$$

The expressions (B.1), (B.3) should be substituted into (3.10), what gives us the linearized form $\widetilde{\mathcal{W}}_{j ; k}$ (5.4).

The summation of the Lagrange series leads to the replacement of the sums by integrals over the original contour $\Gamma_{j ; k}$

$$
\begin{aligned}
& \sum_{k=1}^{m} \epsilon_{k} g_{\sigma}^{(1)}\left(w, \lambda_{k}\right) \hookrightarrow \int_{\Gamma_{\boldsymbol{j} ; \boldsymbol{k}}} g_{\sigma}^{(1)}(w, \lambda) z_{\boldsymbol{j} ; \boldsymbol{k}}(\lambda) d \lambda=-L_{\Gamma_{\boldsymbol{j} ; \boldsymbol{k}}}\left[z_{\boldsymbol{j} ; \boldsymbol{k}}\right](w+i \sigma c), \\
& \sum_{k=1}^{m} \sum_{k^{\prime}=1}^{m} \epsilon_{k} \epsilon_{k^{\prime}} g^{(2)}\left(\lambda_{k}, \lambda_{k^{\prime}}\right) \hookrightarrow \int_{\Gamma_{\boldsymbol{j} ; \boldsymbol{k}}} g^{(2)}(\lambda, \mu) z_{\boldsymbol{j} ; \boldsymbol{k}}(\lambda) z_{\boldsymbol{j} ; \boldsymbol{k}}(\mu) d \lambda d \mu=-C_{0}\left[z_{\boldsymbol{j} ; \boldsymbol{k}}, \Gamma_{\boldsymbol{j} ; \boldsymbol{k}}\right],
\end{aligned}
$$

where $z_{\boldsymbol{j} ; \boldsymbol{k}}(\lambda)$ solves the integral equation (5.8), $L_{\Gamma_{\boldsymbol{j} ; \boldsymbol{k}}}$ is the operator of Cauchy transform (4.14), and for any contour $\mathcal{L}$

$$
C_{0}\left[z_{\boldsymbol{j} ; \boldsymbol{k}}, \mathcal{L}\right]=\int_{\mathcal{L}} \frac{z_{\boldsymbol{j} ; \boldsymbol{k}}(\lambda) z_{\boldsymbol{j} ; \boldsymbol{k}}(\mu)}{(\lambda-\mu-i c)^{2}} d \lambda d \mu
$$


Substituting these formulae into 3.10 and moving the contours from $\Gamma_{\boldsymbol{j} ; \boldsymbol{k}}$ to $\hat{\mathcal{C}}_{\boldsymbol{j} ; \boldsymbol{k}}$ we get that all the explicit dependence on the poles $r_{\boldsymbol{j} / \boldsymbol{k}}^{ \pm}$cancels out and

$$
\begin{aligned}
\widetilde{\mathcal{W}}_{\boldsymbol{j} ; \boldsymbol{k}}\left(\int_{\Gamma_{\boldsymbol{j} ; \boldsymbol{k}}} g_{\sigma}^{(1)}(\lambda)\right. & \left.z_{\boldsymbol{j} ; \boldsymbol{k}}(\lambda) d \lambda ; \int_{\Gamma_{\boldsymbol{j} ; \boldsymbol{k}}} g^{(2)}(\lambda, \mu) z_{\boldsymbol{j} ; \boldsymbol{k}}(\lambda) z_{\boldsymbol{j} ; \boldsymbol{k}}(\mu) d \lambda d \mu\right) \\
=\widetilde{\mathcal{W}} & \left(\int_{\hat{\mathcal{C}}_{\boldsymbol{j} ; \boldsymbol{k}}} g_{\sigma}^{(1)}(\lambda) z_{\boldsymbol{j} ; \boldsymbol{k}}(\lambda) d \lambda ; \int_{\hat{\mathcal{C}}_{\boldsymbol{j} ; \boldsymbol{k}}} g^{(2)}(\lambda, \mu) z_{\boldsymbol{j} ; \boldsymbol{k}}(\lambda) z_{\boldsymbol{j} ; \boldsymbol{k}}(\mu) d \lambda d \mu\right),
\end{aligned}
$$

where

$$
\begin{aligned}
& \widetilde{\mathcal{W}}\left(\left\{\int_{\hat{\mathcal{C}}_{\boldsymbol{j} ; \boldsymbol{k}}} g^{(1)}(\lambda) z_{\boldsymbol{j} ; \boldsymbol{k}}(\lambda) d \omega\right\} ; \int_{\hat{\mathcal{C}}_{\boldsymbol{j} ; \boldsymbol{k}}} g^{(2)}(\lambda, \mu) z_{\boldsymbol{j} ; \boldsymbol{k}}(\lambda) z_{\boldsymbol{j} ; \boldsymbol{k}}(\mu) d \lambda d \mu\right) \\
& =\frac{e^{-C_{0}\left[z_{\boldsymbol{j} ; \boldsymbol{k}}, \hat{\mathcal{C}}_{\boldsymbol{j} ; \boldsymbol{k}}\right]}\left(e^{2 \pi i \alpha}-1\right)^{2} \operatorname{det}\left(I+\frac{1}{2 \pi i} \hat{U}^{(1)}\left[z_{\boldsymbol{j} ; \boldsymbol{k}}\right]\right) \operatorname{det}\left(I+\frac{1}{2 \pi i} \hat{U}^{(2)}\left[z_{\boldsymbol{j} ; \boldsymbol{k}}\right]\right)}{\left[e^{L_{\hat{\boldsymbol{j}} ; \boldsymbol{k}}\left[z_{\boldsymbol{j} ; \boldsymbol{k}}\right]\left(\theta_{1}+i c\right)}-e^{2 \pi i \alpha+L_{\hat{\mathcal{C}}_{\boldsymbol{j} ; \boldsymbol{k}}}\left[z_{\boldsymbol{j} ; \boldsymbol{k}}\right]\left(\theta_{1}-i c\right)}\right]\left[e^{-L_{\hat{\mathcal{j}}_{\boldsymbol{j} ; \boldsymbol{k}}}\left[z_{\boldsymbol{j} ; \boldsymbol{k}}\right]\left(\theta_{2}-i c\right)}-e^{2 \pi i \alpha-L_{\hat{\mathcal{C}} \boldsymbol{j} \boldsymbol{k}}\left[z_{\boldsymbol{j} ; \boldsymbol{k}}\right]\left(\theta_{2}+i c\right)}\right]},
\end{aligned}
$$

and the kernels of the integral operators $\hat{U}^{(1)}\left(w, w^{\prime},\left[z_{\boldsymbol{j} ; \boldsymbol{k}}\right]\right)$ and $\hat{U}^{(2)}\left(w, w^{\prime},\left[z_{\boldsymbol{j} ; \boldsymbol{k}}\right]\right)$ have the form

$$
\begin{gathered}
\hat{U}^{(1)}\left(w, w^{\prime},\left[z_{\boldsymbol{j} ; \boldsymbol{k}}\right]\right)=-e^{L_{\hat{\mathcal{j}}_{\boldsymbol{j} ; \boldsymbol{k}}}\left[z_{\boldsymbol{j} ; \boldsymbol{k}}\right](w)} \cdot \frac{K_{\alpha}\left(w-w^{\prime}\right)-K_{\alpha}\left(\theta_{1}-w^{\prime}\right)}{e^{L_{\hat{\mathcal{C}}_{\boldsymbol{j} ; \boldsymbol{k}}}\left[z_{\boldsymbol{j} ; \boldsymbol{k}}\right](w+i c)}-e^{2 \pi i \alpha+L_{\hat{\mathcal{C}}_{\boldsymbol{j} ; \boldsymbol{k}}}\left[z_{\boldsymbol{j} ; \boldsymbol{k}}\right](w-i c)}}, \\
\hat{U}^{(2)}\left(w, w^{\prime},\left[z_{\boldsymbol{j} ; \boldsymbol{k}}\right]\right)=e^{-L_{\hat{\mathcal{C}}_{\boldsymbol{j} ; \boldsymbol{k}}\left[z_{\boldsymbol{j} ; \boldsymbol{k}}\right]\left(w^{\prime}\right)}} \cdot \frac{K_{\alpha}\left(w-w^{\prime}\right)-K_{\alpha}\left(w-\theta_{2}\right)}{e^{-L_{\hat{\mathcal{C}}_{\boldsymbol{j} ; \boldsymbol{k}}}\left[z_{\boldsymbol{j} ; \boldsymbol{k}}\right]\left(w^{\prime}-i c\right)}-e^{2 \pi i \alpha-L_{\hat{\mathcal{C}}_{\boldsymbol{j} ; \boldsymbol{k}}}\left[z_{\boldsymbol{j} ; \boldsymbol{k}}\right]\left(w^{\prime}+i c\right)}} .
\end{gathered}
$$

Both integral operators act on a counterclockwise oriented closed contour surrounding the contour $\hat{\mathcal{C}}_{\boldsymbol{j} ; \boldsymbol{k}}$.

\section{Deformation of the contours $\mathcal{C}_{j ; k}$}

In this section we calculate the difference $\mathcal{A}_{\mathcal{C}_{j ; k}}([g],[\nu])-\mathcal{A}_{\Gamma_{j ; k}}([g],[\nu])$ (see 4.11)). We shall consider some fixed contours $\mathcal{C}_{\boldsymbol{j} ; \boldsymbol{k}}$ and $\Gamma_{\boldsymbol{j} ; \boldsymbol{k}}$. Hence, for brevity, we omit the subscripts $\boldsymbol{j} ; \boldsymbol{k}$ in the following. Moreover, it is clear that, without loss of any generality, we can set $\boldsymbol{j}=(1, \ldots, n)$ and $\boldsymbol{k}=(1, \ldots, n)$. Also we note that we can carry out the intermediate computations up to integer multiples of $2 i \pi$. This is justified in as much as we take the exponential at the end.

Observe that the contour $\mathcal{C} \cup-\Gamma$ surrounds the points $r_{k}^{+}$in the counterclockwise direction and the points $r_{k}^{-}$in the clockwise direction. Therefore if $f(\omega)$ is holomorphic in a domain containing $\Gamma$ and $\mathcal{C}$, then

$$
\int_{\Gamma} f^{\prime}(\omega) \nu(\omega) d \omega=\int_{\mathcal{C}} f^{\prime}(\omega) \nu(\omega) d \omega+\sum_{k=1}^{n}\left(f\left(r_{k}^{-}\right)-f\left(r_{k}^{+}\right)\right) .
$$


Using (C.1) one can easily calculate the difference of single integrals entering (4.11):

$$
-\int_{\mathcal{C}}\left(i x+g^{\prime}(\lambda)\right) \nu(\lambda) d \lambda+\int_{\Gamma}\left(i x+g^{\prime}(\lambda)\right) \nu(\lambda) d \lambda=\sum_{k=1}^{n}\left(i x\left(r_{k}^{-}-r_{k}^{+}\right)+g\left(r_{k}^{-}\right)-g\left(r_{k}^{+}\right)\right) \text {. }
$$

The calculation of the difference of the double integrals in 4.11) is more involved. It is convenient to present $\nu(\lambda)$ in the form

$$
\nu(\lambda)=\tilde{\nu}(\lambda)-\frac{1}{2 \pi i} \log \left\{\prod_{k=1}^{n} \frac{\left(\lambda-s_{k}^{+}\right)\left(\lambda-s_{k}^{-}\right)}{\left(\lambda-r_{k}^{+}\right)\left(\lambda-r_{k}^{-}\right)}\right\},
$$

where $\tilde{\nu}(\lambda)$ is holomorphic in a domain containing both contours $\mathcal{C}$ and $\Gamma$. Let

$$
J(\mathcal{C})=\iint_{\mathcal{C}} \frac{\nu(\lambda) \nu(\mu)}{\left(\lambda-\mu_{+}\right)^{2}} d \lambda d \mu, \quad J(\Gamma)=\iint_{\Gamma} \frac{\nu(\lambda) \nu(\mu)}{\left(\lambda-\mu_{+}\right)^{2}} d \lambda d \mu .
$$

Let us also introduce auxiliary functions

$$
\alpha_{+}(\lambda ; \mathcal{C})=\log \left\{\prod_{k=1}^{n} \frac{\lambda-s_{k}^{+}}{\lambda-r_{k}^{-}}\right\}, \quad \alpha_{-}(\lambda ; \mathcal{C})=\log \left\{\prod_{k=1}^{n} \frac{\lambda-s_{k}^{-}}{\lambda-r_{k}^{+}}\right\},
$$

and

$$
\alpha_{+}(\lambda ; \Gamma)=\log \left\{\prod_{k=1}^{n} \frac{\left(\lambda-s_{k}^{+}\right)}{\left(\lambda-r_{k}^{+}\right)}\right\}, \quad \alpha_{-}(\lambda ; \Gamma)=\log \left\{\prod_{k=1}^{n} \frac{\left(\lambda-s_{k}^{-}\right)}{\left(\lambda-r_{k}^{-}\right)}\right\} .
$$

It is easy to see that the functions $\alpha_{ \pm}(\lambda ; \mathcal{C})$ are holomorphic to the left (resp. to the right) from the contour $\mathcal{C}$. Similarly the functions $\alpha_{ \pm}(\lambda ; \Gamma)$ are holomorphic to the left (resp. to the right) from the contour $\Gamma$. All the functions (C.5) and (C.6) behave as $O\left(\lambda^{-1}\right)$ when $\lambda \rightarrow \infty$, and

$$
\alpha_{+}(\lambda ; \mathcal{C})+\alpha_{-}(\lambda ; \mathcal{C})=\alpha_{+}(\lambda ; \Gamma)+\alpha_{-}(\lambda ; \Gamma)=\log \left\{\prod_{k=1}^{n} \frac{\left(\lambda-s_{k}^{+}\right)\left(\lambda-s_{k}^{-}\right)}{\left(\lambda-r_{k}^{+}\right)\left(\lambda-r_{k}^{-}\right)}\right\} .
$$

Using C.3 and C.5 we can present $J(\mathcal{C})$ as a sum of four integrals $J(\mathcal{C})=\sum_{j=1}^{4} J_{j}(\mathcal{C})$, where

$$
\begin{aligned}
& J_{1}(\mathcal{C})=\iint_{\mathcal{C}} \frac{\tilde{\nu}(\lambda) \tilde{\nu}(\mu)}{\left(\lambda-\mu_{+}\right)^{2}} d \lambda d \mu, \\
& J_{2}(\mathcal{C})=-\frac{1}{2 \pi i} \iint_{\mathcal{C}} \frac{\tilde{\nu}(\lambda)\left(\alpha_{+}(\mu ; \mathcal{C})+\alpha_{-}(\mu ; \mathcal{C})\right)}{\left(\lambda-\mu_{+}\right)^{2}} d \lambda d \mu, \\
& J_{3}(\mathcal{C})=-\frac{1}{2 \pi i} \iint_{\mathcal{C}} \frac{\left(\alpha_{+}(\lambda ; \mathcal{C})+\alpha_{-}(\lambda ; \mathcal{C})\right) \tilde{\nu}(\mu)}{\left(\lambda-\mu_{+}\right)^{2}} d \lambda d \mu, \\
& J_{4}(\mathcal{C})=\frac{1}{(2 \pi i)^{2}} \iint_{\mathcal{C}} \frac{\left(\alpha_{+}(\lambda ; \mathcal{C})+\alpha_{-}(\lambda ; \mathcal{C})\right)\left(\alpha_{+}(\mu ; \mathcal{C})+\alpha_{-}(\mu ; \mathcal{C})\right)}{\left(\lambda-\mu_{+}\right)^{2}} d \lambda d \mu .
\end{aligned}
$$


Similarly $J(\Gamma)=\sum_{j=1}^{4} J_{j}(\Gamma)$ where $J_{j}(\Gamma)$ is obtained from $J_{j}(\mathcal{C})$ by replacing everywhere $\mathcal{C}$ by $\Gamma$ in C.8 C.11.

Since $\tilde{\nu}(\lambda)$ is holomorphic in a domain containing the contours $\mathcal{C}$ and $\Gamma$, we conclude that $J_{1}(\mathcal{C})-J_{1}(\Gamma)=0$. The integrals $J_{4}(\mathcal{C})$ and $J_{4}(\Gamma)$ can be taken explicitly. For example, using the analytic properties of $\alpha_{ \pm}(\lambda ; \mathcal{C})$ we have

$$
\begin{aligned}
& J_{4}(\mathcal{C})=\frac{1}{2 \pi i} \int_{\mathcal{C}} \alpha_{+}^{\prime}(\mu ; \mathcal{C})\left(\alpha_{+}(\mu ; \mathcal{C})+\alpha_{-}(\mu ; \mathcal{C})\right) d \mu=\frac{1}{2 \pi i} \int_{\mathcal{C}} \alpha_{+}^{\prime}(\mu ; \mathcal{C}) \alpha_{-}(\mu ; \mathcal{C}) d \mu \\
& =\frac{1}{2 \pi i} \int_{\mathcal{C}} \sum_{k=1}^{n}\left(\frac{1}{\mu-s_{k}^{+}}-\frac{1}{\mu-r_{k}^{-}}\right) \alpha_{-}(\mu ; \mathcal{C}) d \mu=\log \left\{\prod_{j, k=1}^{n}\left(\frac{r_{k}^{-}-s_{j}^{-}}{r_{k}^{-}-r_{j}^{+}} \cdot \frac{s_{k}^{+}-r_{j}^{+}}{s_{k}^{+}-s_{j}^{-}}\right)\right\} .
\end{aligned}
$$

The expression for $J_{4}(\Gamma)$ is obtained from $\mathrm{C.12}$ via the replacement $r^{+} \leftrightarrow r^{-}$. Then we have

$$
J_{4}(\mathcal{C})-J_{4}(\Gamma)=\log \left\{\prod_{j, k=1}^{n}\left(\frac{r_{k}^{-}-s_{j}^{-}}{r_{k}^{-}-r_{j}^{+}} \cdot \frac{s_{k}^{+}-r_{j}^{+}}{s_{k}^{+}-r_{j}^{-}} \cdot \frac{r_{k}^{+}-r_{j}^{-}}{r_{k}^{+}-s_{j}^{-}}\right)\right\} .
$$

Calculating $J_{2}$ and $J_{3}$ we can take explicitly only one of two integrals. We have

$$
J_{2}(\mathcal{C})=\int_{\mathcal{C}} \alpha_{-}^{\prime}(\lambda ; \mathcal{C}) \tilde{\nu}(\lambda) d \lambda=\int_{\mathcal{C}} \sum_{k=1}^{n}\left(\frac{1}{\lambda-s_{k}^{-}}-\frac{1}{\lambda-r_{k}^{+}}\right) \tilde{\nu}(\lambda) d \lambda,
$$

and

$$
J_{3}(\mathcal{C})=-\int_{\mathcal{C}} \alpha_{+}^{\prime}(\mu ; \mathcal{C}) \tilde{\nu}(\mu) d \mu=\int_{\mathcal{C}} \sum_{k=1}^{n}\left(\frac{1}{\mu-r_{k}^{-}}-\frac{1}{\mu-s_{k}^{+}}\right) \tilde{\nu}(\mu) d \mu .
$$

The expressions for $J_{2}(\Gamma)$ and $J_{3}(\Gamma)$ can be obtained from C.14 and C.15) via the replacements $\mathcal{C} \rightarrow \Gamma$ and $r^{+} \leftrightarrow r^{-}$. Then we have

$$
\begin{aligned}
\sum_{s=2,3}\left(J_{s}(\mathcal{C})-J_{s}(\Gamma)\right)= & \int_{\mathcal{C} \cup-\Gamma} \sum_{k=1}^{n}\left(\frac{1}{\lambda-s_{k}^{-}}-\frac{1}{\lambda-s_{k}^{+}}\right) \tilde{\nu}(\lambda) d \lambda \\
& \quad+\int_{\mathcal{C} \cup \Gamma} \sum_{k=1}^{n}\left(\frac{1}{\lambda-r_{k}^{-}}-\frac{1}{\lambda-r_{k}^{+}}\right) \tilde{\nu}(\lambda) d \lambda .
\end{aligned}
$$

The integral in the first line of (C.16) vanishes as the contour $\mathcal{C} \cup-\Gamma$ does not surround the points $s_{k}^{ \pm}$. The second integral in (C.16) gives

$$
\sum_{s=2,3}\left(J_{s}(\mathcal{C})-J_{s}(\Gamma)\right)=2 \int_{\Gamma} \sum_{k=1}^{n}\left(\frac{1}{\lambda-r_{k}^{-}}-\frac{1}{\lambda-r_{k}^{+}}\right) \tilde{\nu}(\lambda) d \lambda-2 \pi i \sum_{k=1}^{n}\left(\tilde{\nu}\left(r_{k}^{+}\right)+\tilde{\nu}\left(r_{k}^{-}\right)\right)
$$


Here we have used that the contour $\mathcal{C} \cup-\Gamma$ surrounds the points $r_{k}^{+}$in the counterclockwise direction and the points $r_{k}^{-}$in the clockwise direction. Substituting into C.17 the function $\tilde{\nu}(\lambda)$ in terms of $\nu(\lambda)$ via (C.3) we obtain after simple algebra

$$
\begin{aligned}
\sum_{s=2,3}\left(J_{s}(\mathcal{C})-J_{s}(\Gamma)\right)=\sum_{k=1}^{n}\left(2 L_{\Gamma}[\nu]\left(r_{k}^{-}\right)-2 L_{\Gamma}[\nu]\left(r_{k}^{+}\right)-2 \pi i \tilde{\nu}\left(r_{k}^{+}\right)-2 \pi i \tilde{\nu}\left(r_{k}^{-}\right)\right) & \\
& +2 \log \prod_{j, k=1}^{n}\left(\frac{r_{k}^{-}-s_{j}^{+}}{r_{k}^{-}-r_{j}^{+}} \cdot \frac{r_{k}^{+}-s_{j}^{-}}{r_{k}^{+}-r_{j}^{-}}\right)
\end{aligned}
$$

where we have used the Cauchy transform over the contour $\Gamma$ of the function $\nu(\lambda)$ (see (4.14)).

It remains to find $\tilde{\nu}\left(r_{k}^{ \pm}\right)$. These numbers can be expressed in terms of the residues of the Fermi weight $\vartheta_{\text {reg }}\left(r_{k}^{ \pm}\right)$in the points $r_{k}^{ \pm}$. We have

$$
1+\gamma \vartheta(\lambda) F(\lambda)=e^{-2 \pi i \nu(\lambda)}=e^{-2 \pi i \tilde{\nu}(\lambda)} \prod_{j=1}^{n} \frac{\left(\lambda-s_{j}^{+}\right)\left(\lambda-s_{j}^{-}\right)}{\left(\lambda-r_{j}^{+}\right)\left(\lambda-r_{j}^{-}\right)} .
$$

Hence,

$$
\gamma \vartheta_{r e g}\left(r_{k}^{ \pm}\right) F\left(r_{k}^{ \pm}\right)=e^{-2 \pi i \tilde{\nu}\left(r_{k}^{ \pm}\right)} \prod_{j=1}^{n} \frac{\left(r_{k}^{ \pm}-s_{j}^{+}\right)\left(r_{k}^{ \pm}-s_{j}^{-}\right)}{\left(r_{k}^{ \pm}-r_{j}^{\mp}\right)} \prod_{\substack{j=1 \\ j \neq k}}^{n} \frac{1}{r_{k}^{ \pm}-r_{j}^{ \pm}} .
$$

Thus, combining (C.13), (C.18), and (C.20) we obtain

$$
e^{J(\mathcal{C})-J(\Gamma)}=\left(\operatorname{det}_{n} \frac{1}{r_{j}^{+}-r_{k}^{-}}\right)^{2} \prod_{k=1}^{n}\left[\gamma^{2} e^{2 L_{\Gamma}[\nu]\left(r_{k}^{-}\right)-2 L_{\Gamma}[\nu]\left(r_{k}^{+}\right)} \vartheta_{r e g}\left(r_{k}^{+}\right) \vartheta_{r e g}\left(r_{k}^{-}\right) F\left(r_{k}^{+}\right) F\left(r_{k}^{-}\right)\right],
$$

where we have used

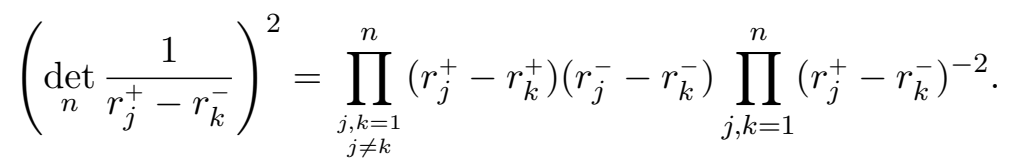

Taking into account (C.2) we immediately arrive at the formulae 4.16, 4.17).

We insist that all the above computations have been done by using the sole pole/zero structure of $e^{-2 i \pi \nu(\omega)}$. They are thus valid in the specific case where one considers $\nu=z_{\boldsymbol{j} ; \boldsymbol{k}}$.

\section{Continuous generalization of the multiple Lagrange series}

The continuous generalization of the multiple Lagrange series has the form

$$
\hat{G}=\left.\sum_{n=0}^{\infty} \frac{1}{n !} \int_{\mathcal{L}} d^{n} \lambda \prod_{j=1}^{n} \frac{\partial}{\partial \epsilon_{j}} \prod_{j=1}^{n} f\left(\sum_{a=1}^{n} \epsilon_{a} \xi\left(\lambda_{a}, \lambda_{j}\right)\right) \cdot F\left(\sum_{a=1}^{n} h_{1}\left(\lambda_{a}\right) \epsilon_{a} ; \ldots,\right)\right|_{\epsilon_{j}=0} .
$$


Here the integrals are taken over some contour $\mathcal{L}$, the functions $f, F, \xi$, and $h_{1}$ are holomorphic the corresponding neighborhoods. The argument of the function $F$ may also contain double sums $\sum_{a, b} h_{2}\left(\lambda_{a}, \lambda_{b}\right) \epsilon_{a} \epsilon_{b}$ etc, like, for example, in (5.4). Apart from obvious modifications, the presence of such multiple sums does not affect the result. Hence, we have omitted these arguments of the function $F$ for brevity.

This series was studied in [1]. If there exists a $R_{0}>0$ such that

$$
\sup _{\phi \in[0 ; 2 \pi]} \sup _{\mu \in \mathcal{L}}\left|f\left(R_{0} e^{i \phi} \int_{\mathcal{L}}|\xi(\lambda, \mu)| d \lambda\right)\right|<R_{0},
$$

then it is absolutely convergent. In such a case, the result of the summation reads

$$
\hat{G}=\frac{F\left(\int_{\mathcal{L}} h_{1}(\mu) z(\mu) d \mu ; \ldots\right)}{\operatorname{det}_{\mathcal{L}}\left[\delta(\lambda-\mu)-\xi(\mu, \lambda) f^{\prime}\left(\int_{\mathcal{L}} \xi(\nu, \lambda) z(\nu) d \nu\right)\right]},
$$

where the function $z(\mu)$ is the unique solution to the integral equation

$$
z(\mu)=f\left(\int_{\mathcal{L}} \xi(\lambda, \mu) z(\lambda) d \lambda\right) .
$$

The denominator of (D.3) contains the Fredholm determinant of a linear integral operator acting on $\mathcal{L}$. If the function $F$ depends on multiple sums, then these should be replaced by multiple integrals, like, for example,

$$
\sum_{a, b=1}^{n} h_{2}\left(\lambda_{a}, \lambda_{b}\right) \epsilon_{a} \epsilon_{b} \hookrightarrow \int_{\mathcal{L}} h_{2}(\lambda, \mu) z(\lambda) z(\mu) d \lambda d \mu,
$$

etc. It is important to note that the form of the integral equation $(\mathrm{D.4})$ does not depend on the function $F$.

The result (D.3) can be directly applied to the summation of the series (5.5), where the integration contour $\mathcal{L}$ coincides with $\Gamma_{\boldsymbol{j} ; \boldsymbol{k}}$ and the function $f$ is $\phi_{\boldsymbol{j} ; \boldsymbol{k}}$ (5.3).

\section{References}

[1] N. Kitanine, K. K. Kozlowski, J. M. Maillet, N. A. Slavnov, V. Terras, J. Stat. Mech. 04 2009 (2009) P04003.

[2] N. Kitanine, J. M. Maillet, and V. Terras, Nucl. Phys. B567 (2000) 554.

[3] N. Kitanine, J. M. Maillet, N. Slavnov, and V. Terras, Nucl. Phys. B641 (2002) 487.

[4] N. Kitanine, J. M. Maillet, N. A. Slavnov, and V. Terras, Nucl. Phys. B712 (2005) 600. 
[5] N. Kitanine, J. M. Maillet, N. A. Slavnov, and V. Terras, In Solvable Lattice Models $2004-$ Recent Progress on Solvable Lattice Models, volume 1480 of Kokyuroku. RIMS, Kyoto, 2006.

[6] N. Kitanine, K. K. Kozlowski, J. M. Maillet, N. A. Slavnov, and V. Terras, J. Stat. Mech. Theory Exp. (2007) P01022.

[7] E. H. Lieb and W. Liniger, Phys. Rev. 130 (1963), 1605.

[8] E. Lieb and D. Mattis (eds.), Mathematical Physics in One Dimension, New York: Academic Press, (1966).

[9] M. Girardeau, J. Math. Phys. 1 (1960) 516.

[10] L. D. Faddeev, E. K. Sklyanin and L. A. Takhtajan, Theor. Math. Phys. 40 (1980) 688.

[11] L.D. Faddeev, in: Les Houches Lectures Quantum Symmetries, eds A. Connes et al, (North Holland 1998), 149.

[12] V. E. Korepin, N. M. Bogoliubov, A. G. Izergin, Quantum Inverse Scattering Method and Correlation Functions, Cambridge University Press, 1993.

[13] C. N. Yang and C. P. Yang, J. Math. Phys., 10 (1969) 1115.

[14] T. C. Dorlas, J. T. Lewis and J. V. Pulé, Comm. Math. Phys. 124 (1989) 365.

[15] A. Lenard, J. Math. Phys. 7 (1966), 1268.

[16] V. E. Korepin, Commun. Math. Phys. 113 (1987) 177.

[17] V. E. Korepin and N. A. Slavnov, Comm. Math.Phys. 129 (1990), 103.

[18] A.R. Its, A.G. Izergin, and V.E. Korepin, Commun. Math. Phys. 129 (1990) 205.

[19] A.R. Its, A.G. Izergin, and V.E. Korepin, Commun. Math. Phys. 130 (1990) 471.

[20] A. R. Its, A. G. Izergin and V. E. Korepin and G. G. Varguzin, Physica D54 (1991), 351.

[21] N. M. Bogoliubov, V. E. Korepin, Theor. Math. Phys. 60 iss.2 (1984) 808.

[22] V.E. Korepin, Funct. Anal. and Appl. 23 (1989) 12.

[23] V. E. Korepin and N. A. Slavnov, Comm. Math.Phys. 136 (1991) 633.

[24] T. Kojima, V. E. Korepin and N. A. Slavnov, Comm. Math.Phys. 188 (1997), 657.

[25] A. R. Its and N. A. Slavnov, Theor. Math. Phys. 119, iss.2 (1999) 541.

[26] N.A. Slavnov, Theor. Math. Phys. 121 iss.1 (1999) 117. 
[27] V. N. Popov, Functional Integrals in Quantum Field Theory and Statistical Physics, D. Reidel, Dordrecht, 1983.

[28] A.A. Belavin, A.M. Polyakov and A.B. Zamolodchikov, Nucl. Phys. B241 (1984) 333.

[29] J. L. Cardy, J. Phys. A: Math. Gen. 17 (1984) L385.

[30] J. L. Cardy, Nucl. Phys. B270 (1986) 186.

[31] J. L. Cardy, "Scaling and renormalization in statistical physics", Cambridge Lecture Notes in Physics, 1996.

[32] A. Seel, T. Bhattacharyya, F. Göhmann, A. Klümper, Journ. Stat. Mech. (2007) P08030.

[33] A. Klümper, Lecture Notes in Physics, 645 (2004) 349.

[34] M. Takahashi, M. Shiroishi, A. Klümper J. Phys. A : Math. Gen. 34 (2001) L187.

[35] N. M. Bogoliubov, A.G. Izergin and V. E. Korepin, Lecture Notes in Physics, 242 (1985) 221.

[36] A. Klümper, M. Batchelor, and P. Pearce, J. Phys. A: Math. Gen. 24 (1991) 3111.

[37] A. Klümper, Z. Phys. B91 (1993) 507.

[38] A. Klümper, T. Wehner, and J. Zittartz, J. Phys. A : Math. Gen. 26 (1993) 2815.

[39] N. M. Bogoliubov, V. E. Korepin, Theor. Math. Phys. 64 iss.1 (1985) 708.

[40] N .M. Bogoliubov and V. E. Korepin, Nucl. Phys. B257, (1985), 766.

[41] V. E. Korepin, N.A. Slavnov, Theor. Math. Phys., 68 iss.3 (1986) 955.

[42] P. Dorey and R. Tateo, Nucl. Phys. B482 (1996) 639.

[43] N. Kitanine, K. K. Kozlowski, J. M. Maillet, N. A. Slavnov, V. Terras, Commun. Math. Phys. 291 (2009) 691.

[44] N. A. Slavnov, Theor. Math. Phys. 165 (2010) 1262.

[45] E. T. Whittaker, G. N. Watson, A Course of Modern Analysis, Cambridge University Press, 1927.

[46] I. A. Aizenberg and A. P. Yuzhakov, Integral representations and residues in multidimensional complex analysis, AM. Math. Soc, Grad. Text Math, 58, 1978.

[47] K. K. Kozlowski, J. M. Maillet, N. A. Slavnov, "Density-density correlation function of one-dimensional bosons at low temperature.", to appear. 